\title{
STRATEGIES IN USING NUMERICAL WAVE MODELS IN OCEAN/COASTAL APPLICATIONS
}

\author{
Eugen Rusu \\ Department of Applied Mechanics, Galati University 'Dunarea de Jos', Romania., erusu@ugal.ro
}

Follow this and additional works at: https://jmstt.ntou.edu.tw/journal

Part of the Engineering Commons

\section{Recommended Citation}

Rusu, Eugen (2011) "STRATEGIES IN USING NUMERICAL WAVE MODELS IN OCEAN/COASTAL APPLICATIONS," Journal of Marine Science and Technology. Vol. 19: Iss. 1, Article 8.

DOI: 10.51400/2709-6998.2138

Available at: https://jmstt.ntou.edu.tw/journal/vol19/iss1/8

This Research Article is brought to you for free and open access by Journal of Marine Science and Technology. It has been accepted for inclusion in Journal of Marine Science and Technology by an authorized editor of Journal of Marine Science and Technology. 


\title{
STRATEGIES IN USING NUMERICAL WAVE MODELS IN OCEAN/COASTAL APPLICATIONS
}

\author{
Eugen Rusu*
}

\begin{abstract}
Key words: wind waves, spectral wave models, ocean and coastal environment, computational strategies, wave predictions.
\end{abstract}

\begin{abstract}
The objective of the present work is to illustrate the performances of the numerical wave models in ocean and coastal environment. Third generation wave models are considered nowadays the most appropriate for such task. These are full spectral models based on the integration on the wave energy (or alternatively wave action) balance equation. In order to cover more aspects related with the modelling process hindcast, nowcast and forecast schemes are discussed and illustrated along six case studies. The major model used was SWAN (acronym for Simulating Waves Nearshore) which is a very flexible model that can be applied in a wide range of coastal applications being effective from high resolution coastal areas up to quasi oceanic scales. In both hindcasts and forecasts the wave forcing was provided by generation models (WAM and WW3), while in nowcast schemes buoy data were used. Various coastal environments that are rather different from the point of view of the bathymetric features and of the characteristics of the environmental matrix were considered. These are the Portuguese continental nearshore with higher resolution sub domains, Madeira Archipelago, the nearshore of Sardinia Island in the Mediterranean Sea and the Black Sea. A general conclusion of this work would be that, despite some limitations, the wave models provide an effective framework in predicting wave conditions in ocean and coastal environment.
\end{abstract}

\section{INTRODUCTION}

The irregular nature of wind induces irregular heights and periods to wind generated waves. Due to this specific nature, the sea surface is continually varying, which means that a deterministic approach to describe the sea surface is in general not feasible. On the other hand, statistical properties of the surface, like average wave height, wave periods and directions,

Paper submitted 08/11/09; revised 01/01/10; accepted 10/01/10. Author for correspondence: Eugen Rusu (e-mail: erusu@ugal.ro).

*Department of Applied Mechanics, Galati University 'Dunarea de Jos', Romania. appear to vary slowly in time and space, compared to typical wave periods and wave lengths.

The surface elevation of waves in the ocean, at any location and any time, can be seen as the sum of a large number of harmonic waves, each of which has been generated by turbulent wind in different places and times. They are therefore statistically independent in their origin. Under these conditions, the sea surface elevation on a time scale of one hundred characteristic wave periods is sufficiently well described as a stationary Gaussian process and can be described by the so called random-phase model.

Since a description of ocean waves in the time domain is rather limited, many manipulations are more readily described and understood using the variance density spectrum. This is the Fourier transform of the auto-covariance function of the free surface elevation.

Spectral phase averaging wave models are used nowadays on a wide range to predict the wave conditions at various scales. Over the past two decades, a number of advanced spectral wind-wave models, known as third-generation models, have been developed such as WAM (acronym from Wave Modeling) [33], WW3 (acronym from Wave Watch 3) [31], SWAN [4] and STWAVE (acronym from Steady State Wave Model) [28]. These models solve the spectral action balance equation without any a priori restrictions on the spectrum for the evolution of wave growth.

WAM and WW3 are currently known as the state of the art models for wave generation while SWAN and STWAVE are used mainly as models for wave transformation. The physics for the transformation scale is more elaborated to account better for the processes specific in the coastal environment as refraction, shoaling, breaking or triad wave-wave interactions.

As regards SWAN has to be underlined that in the last versions it goes far beyond the condition of a model only for transformation scale, its range being extended in both offshore and nearshore directions. In relationship with the offshore extension the main improvements are: the high order propagation scheme S\&L [29], almost free of numerical diffusion, that is associated to large scale propagations in the non stationary mode, and the parameterization to counteract the Garden Sprinkler effect [3], that may show up due to this small numerical diffusion associated with a reduced resolution in the spectral space. On the other hand, for extending the model performance in the nearshore direction the most recent improvement concerns in designing a phase decoupled approach 
to account for the diffraction effect [11]. Together with the wave induced set up, diffraction is adequate to local scale simulations and both these two processes are associated mainly with a Cartesian system of coordinates.

Hence, although probably not so efficient from computational point of view for oceanic scales as WW3 or WAM, for the sub oceanic scales SWAN seems to be now the most appropriate wave model. The main reason is its larger flexibility given by including various alternatives for modelling and tuning the physical processes, either in shallow or deep water. In this way, by model calibration a more appropriate combination of the physical processes and parameters can be designed in any particular site, leading to a substantially better quality in the predictions of the main wave parameters.

Hence, the general idea when simulating wave generation and propagation from deep ocean to coastal environment is to nest into the ocean scale models higher resolution models able to account better for the nearshore physical processes. In order to increase gradually the resolution in the models they are also nested in themselves several times.

The concept of nesting implies to compute first the waves on a coarse grid for a larger region and then on a finer grid for a smaller region. The computation on the fine grid uses boundary conditions that are generated by the computation on the coarse grid. Nesting can be repeated on ever decreasing scales using the same type of coordinates for the coarse computations and the nested computations (Cartesian or spherical). Nevertheless, as the present work will also illustrate, sometimes it might be useful at the final level of the coastal model simulations to change the coordinates from spherical to Cartesian and an alternative nesting scheme was designed in this purpose.

The objective of the present work is to present some modeling techniques and computational strategies based on phaseaveraging models. As regards the application of the wave models in complex situations it is also true that there are also relevant cases, as those characterized by strongly varying no homogeneities (bathymetry, currents, etc.) where phase-averaging models should be combined with phase-resolving models, as e.g. examined in [2]. In this case the phase-resolving models should be able to calculate the transformation of irregular wave system over complex bathymetric terrains, as the examples presented and discussed in [6].

\section{THEORETICAL FORMULATIONS}

\section{Energy Balance Equation in Spectral form}

Third generation wave models solve the energy balance Eq. (1) that describes the evolution of the wave spectrum in time, geographical and spectral spaces (the spectral space is usually defined by the relative radian frequency $\sigma$ and the wave direction $\theta$ ):

$$
\frac{D E}{D t}=S,
$$

where: $E$ is the energy density spectrum, $t$ the time and $S$ is the source expressed in terms of energy density.

Nevertheless, most recent versions of the spectral models consider the action density spectrum $(N)$ instead of the energy density spectrum. This is because in the presence of currents action density is conserved whereas energy density is not. The action density is equal to the energy density $(E)$ divided by the relative frequency $(\sigma)$.

If the medium itself is moving with the velocity $\vec{U}$ the frequency of wave passing a field point is shifted due to the Doppler effect:

$$
\omega=\sigma+\vec{k} \cdot \vec{U}=[g k \tanh (k h)]^{1 / 2}+\vec{k} \vec{U}
$$

where $g$ is the acceleration of gravity and $h$ is the water depth. Usually the quantity $\omega$ is called observed or absolute frequency while $\sigma$ is the relative or intrinsic frequency whose functional dependence on $k$ (the wave number in absolute value) is known as the classical dispersion relationship.

\section{Governing Equation in the State of the Art Operational Wave Models}

In this section the governing equations used by the state of the art spectral wave models will be presented and briefly discussed. They are all versions coming from the energy balance Eq. (1).

Since SWAN is the major wave model used in the present study the discussion will start with this model. For large scale applications the governing equation in SWAN is related to the spherical coordinates longitude $(\lambda)$ and latitude $(\varphi)$ :

$$
\frac{\partial N}{\partial t}+\frac{\partial}{\partial \lambda} \dot{\lambda} N+\frac{1}{\cos \varphi} \frac{\partial}{\partial \varphi} \dot{\varphi} N \cos \varphi+\frac{\partial}{\partial \sigma} \dot{\sigma} N+\frac{\partial}{\partial \theta} \dot{\theta} N=\frac{S}{\sigma}
$$

in which:

$$
\left.\begin{array}{l}
\dot{\lambda}=\frac{d \lambda}{d t}=C_{g} \sin \theta(R \cos \varphi)^{-1} \\
\dot{\varphi}=\frac{d \varphi}{d t}=C_{g} R^{-1} \cos \theta \\
\dot{\theta}=\frac{d \theta}{d t}=C_{g} R^{-1} \sin \theta \tan \varphi \\
\dot{\sigma}=c_{\sigma}=\frac{\partial \sigma}{\partial h}\left(\frac{\partial h}{\partial t}+\vec{U} \nabla_{h} h\right)-c_{g} \vec{k} \frac{\partial \vec{U}}{\partial s}
\end{array}\right\}
$$

where $R$ is the radius of the Earth, $\nabla_{h}=\frac{\partial}{\partial x} \vec{i}+\frac{\partial}{\partial y} \vec{j}$ is the horizontal propagation operator and $s$ is the space co-ordinate in the direction of wave propagation. The absolute group velocity $\vec{C}_{g}$ is given by: 


$$
\frac{d \vec{x}}{d t}=\vec{C}_{g}=\frac{\partial \omega}{\partial \vec{k}}=\frac{\partial \sigma}{\partial \vec{k}}+\vec{U}=\vec{c}_{g}+\vec{U}
$$

where $\vec{c}_{g}=\frac{\partial \sigma}{\partial \vec{k}}$ represents the relative group velocity.

For coastal applications the Cartesian coordinates are mostly used in the SWAN simulations and the action balance equation becomes:

$$
\frac{\partial N}{\partial t}+\frac{\partial}{\partial x} \dot{x} N+\frac{\partial}{\partial y} \dot{y} N+\frac{\partial}{\partial \sigma} \dot{\sigma} N+\frac{\partial}{\partial \theta} \dot{\theta} N=\frac{S}{\sigma},
$$

where the propagation velocities of the wave energy in spatial and spectral spaces were defined as: $C_{g x}=\dot{x}, C_{g y}=\dot{y}, C_{\sigma}=\dot{\sigma}$, $C_{\theta}=\dot{\theta}$.

In deep water, three components are significant in the expression of the total source term. They correspond to the atmospheric input $\left(S_{i n}\right)$, whitecapping dissipation $\left(S_{d i s}\right)$ and nonlinear quadruplet interactions $\left(S_{n l}\right)$, respectively. In addition to these three terms, in shallow water physical processes induced by the finite depth effects may play an important role and they correspond to phenomena like bottom friction, depth induced wave breaking and triad nonlinear wave-wave interactions. Hence the total source $(S)$ has the expression:

$$
S=S_{i n}+S_{d i s}+S_{n l}+\underbrace{S_{b f}+S_{b r}+S_{\text {tri }}+\ldots}_{\text {finite depth processes }}
$$

The WAM model computes the evolution of the 2D frequency spectrum $E(\omega, \theta, \lambda, \varphi, t)$. The energy transfer equation used is:

$$
\frac{\partial E}{\partial t}+\frac{\partial}{\partial \lambda}(\dot{\lambda} E)+(\cos \varphi)^{-1} \frac{\partial}{\partial \varphi}(\dot{\varphi} \cos \varphi E)+\frac{\partial}{\partial \theta}(\dot{\theta} E)=S,
$$

The source function $S$ comprises the three basic terms from (7) corresponding to deep water.

In WW3 the governing equation has the following expression:

$$
\frac{\partial N}{\partial t}+\frac{1}{\cos \varphi} \frac{\partial}{\partial \varphi} \dot{\varphi} N \cos \varphi+\frac{\partial}{\partial \lambda} \dot{\lambda} N+\frac{\partial}{\partial k} \dot{k} N+\frac{\partial}{\partial \theta} \dot{\theta}_{g} N=\frac{S}{\sigma},
$$

in which:

$$
\left.\begin{array}{l}
\dot{\lambda}=\frac{d \lambda}{d t}=\frac{c_{g} \sin \theta+U_{\lambda}}{R \cos \varphi}, \dot{\varphi}=\left(c_{g} \cos \theta+U_{\varphi}\right) R^{-1} \\
\dot{\theta}_{g}=\dot{\theta}-\frac{c_{g} \tan \varphi \cos \theta}{R}, \dot{k}=-\frac{\partial \sigma}{\partial h} \frac{\partial h}{\partial s}-\vec{k} \frac{\partial \vec{U}}{\partial s}
\end{array}\right\}
$$

where $U_{\lambda}$ and $U_{\varphi}$ are the components of the current velocity on the longitude and latitude directions, respectively. WW3 can run also in Cartesian coordinates and in this case the governing equation becomes:

$$
\frac{\partial N}{\partial t}+\nabla_{h}[\vec{x} N]+\frac{\partial}{\partial k} \dot{k} N+\frac{\partial}{\partial \theta} \dot{\theta} N=\frac{S}{\sigma} .
$$

Besides the same three source existent in WAM, in WW3 an additional term was introduced to account for bottom friction.

STWAVE model uses the governing equation for steadystate conservation of spectral wave action along a wave ray that is given by [14]:

$$
C_{g x} \frac{\partial}{\partial x} C C_{g} \cos (\mu-\alpha) N+C_{g y} \frac{\partial}{\partial y} C C_{g} \cos (\mu-\alpha) N=\sum \frac{S}{\sigma}
$$

where: $C$ is the phase velocity, $\alpha$ is the wave direction (normal to the wave crest) relative to the $x$-axis, and $\mu$ the direction of the wave ray (relative to the same $x$-axis) defined as:

$$
\mu=\tan ^{-1}\left(\frac{c_{g} \sin \alpha+U \sin \delta}{c_{g} \cos \alpha+U \cos \delta}\right)
$$

with $\delta$ the direction of the current relative to the $x$-axis. Triad wave-wave interactions and bottom friction are not included in STWAVE so that the component of the source term corresponding to finite depth effects ( $S_{f d}$ from Eq. 7) includes only the wave breaking.

At this point some observations can be made. Although all four models discussed above use versions of the energy balance equation some differences can be highlighted only by looking at their governing equations. WAM (cycle 4) solves the balance equation in terms of spectral energy and not spectral action. Moreover, the frequency shifting term is not included in the equation. WW3 solves the action balance equation in the wave number space while SWAN solves the action balance equation in the spectral space. Finally STWAVE solves the action balance equation along the wave ray. It has to be also stressed that the propagation along the wave ray seems to be quite effective for stationary simulations but becomes complicate for the case of the non stationary simulations because of the difficulties that occur in the integration of the source terms if the rays are followed over more than one time step.

\section{Numerical techniques}

All the models discussed above solve a version of the energy balance equation in spectral form with finite difference schemes. The numerical methods are however rather different.

WAM-model solves the wave transport equation explicitly. The source terms and the propagation are computed with different methods and time steps. The source term integration is carried out using a semi implicit integration scheme while the propagation scheme is a first order upwind flux scheme. 
The wind time step can be chosen arbitrarily.

In WW3 the action balance equation is solved using a fractional step method. The first step considers temporal variations of the depth, and corresponding changes in the wave number grid. Other fractional steps consider spatial propagation, intra-spectral propagation and source terms.

In order to cover the wide range of options that are available, both from the point of view of the geographical space covered and also as regards the mode of the simulations performed (stationary or non-stationary), three different numerical schemes were implemented into the SWAN model. These are: the first-order scheme with backward space-backward time (BSBT) that is recommended for small geographical domains in both non-stationary and stationary modes, the second-order scheme with second-order diffusion SORDUP [19] that is recommended for large geographical domains in stationary mode, and the second-order scheme with third-order diffusion S\&L [29] that is recommended for large geographical domains in non-stationary mode.

These numerical schemes have been chosen in SWAN on the basis of robustness, accuracy and economy. Since the nature of the basic equation is such that the state in a grid point is determined by the state in the upwave grid points, the most robust scheme would be an implicit upwind scheme (in both geographic and spectral space). For such a scheme the values of the time and space steps $\Delta t, \Delta x$ and $\Delta y$ would be mutually independent. An implicit scheme is also economical in the sense that such a scheme is unconditionally stable. It permits relatively large time steps in the computations (much larger than for explicit schemes in shallow water).

In STWAVE, the propagation follows the wave rays by applying the conservation of the wave action along backward traced wave rays. Rays are traced in a piecewise manner, from one grid column to the next. The two-dimensional wave spectra are set as input along the first grid column (the offshore boundary). For a point on the second grid column, the spectrum is calculated by back tracing a ray for each frequency and direction component of the spectrum. The ray direction, $\mu$, is determined by Eq. (13). Only ray directions propagating toward the shore $(-87.5$ to $+87.5 \mathrm{deg})$ are included while the energy propagating in the offshore direction is neglected.

\section{WAVE HINDCAST}

\section{Case study 1 - Wave Hindcast in the Iberian Nearshore}

As an attempt to cover a wide range of the features characteristic to the modeling process, six different case studies will be presented and discussed in the next sections. The first two are hindcast studies referring to the west Iberian nearshore and the Black Sea basin, respectively.

The structure of the wave prediction system implemented in the west Iberian coastal environment is suggested in Fig. 1. The WAM model in the improved version that allows for two way nesting [8], covering almost the entire North Atlantic basin, is used for wave generation, whereas SWAN for wave

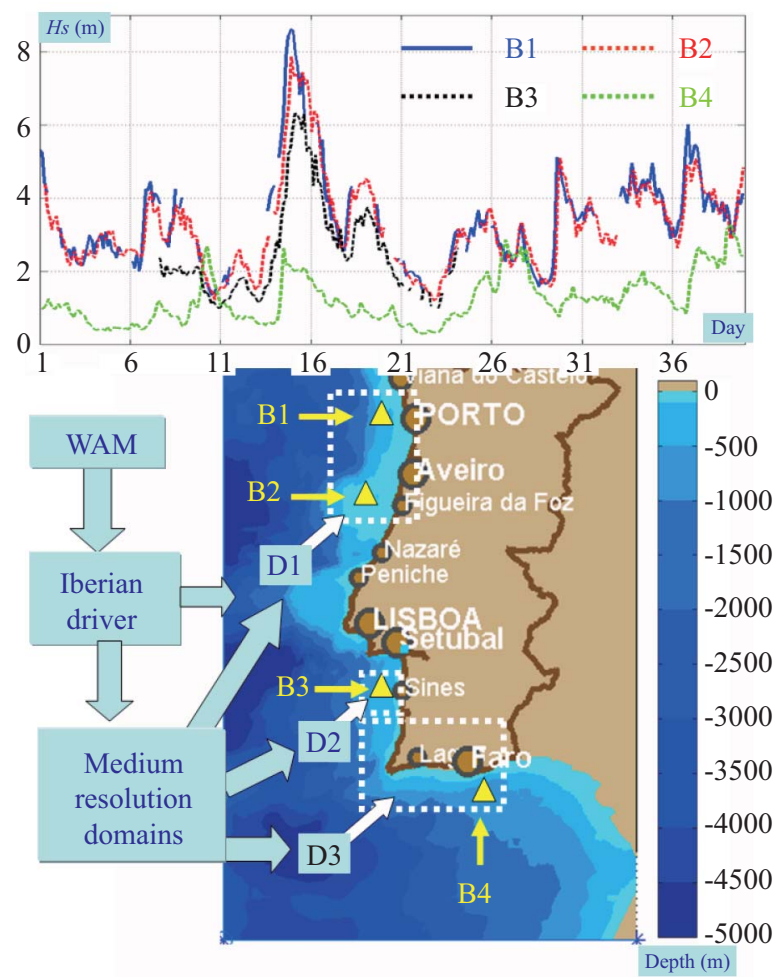

Fig. 1. Case study 1. Diagrammatic scheme suggesting the structure of the wave prediction system used to perform wave hindcast in the west Iberian nearshore; the medium resolution SWAN computational domains considered for model system validation (D1, D2 and D3) are indicated. The locations of the four buoys traditionally operating in the coastal environment of Portugal continental are also illustrated. In the upper side, time variations for the significant wave heights registered for a forty-day period at the four buoys are presented, day 1-1994//01/20- day 40-1994/02/28.

transformation in the coastal environment. As highlighted in the previous sections, recent developments in SWAN (as high order propagation schemes, methods to counteract the Garden-Sprinkler effect, etc.), allow it to be used quite successfully for sub oceanic scales. From this reason, the wave prediction system designed herewith considers a large SWAN domain that covers the entire west Iberian coastal environment and makes the connection between the large scale and the coastal simulations. This domain was denoted in the present work as the west Iberian wave driver and several medium resolution areas are nested inside it. To evaluate the model system reliability, three such domains were defined for the present case and they include all the four buoys considered, as illustrated in Fig. 1. These medium resolution domains were denoted as D1, D2 and D3. The characteristics of the computational grids, for both the west Iberian driver and for the three subsequent domains, are given in Table 1.

Four directional buoys were operating in the Portuguese nearshore and their locations are indicated in Fig. 1. They were denoted as: B1 (Leixões, 9.09W, 41.2N), B2 (Figueira da Foz, 9.24W, 40.26N), B3 (Sines, 8.93W, 37.92N) and B4 
Table 1. Characteristics of the computational domains defined for the wave model simulations. $\Delta x$ and $\Delta y$ represent the resolution in the geographical space, $\Delta t$ - time resolution, $n f$ - number of frequencies in spectral space, $n \theta-$ number of directions in spectral space, ngx - number of grid points in $\mathrm{x}$ direction (longitude), ngy - number of grid points in $y$ direction (latitude) and $\mathrm{np}$ - total number of grid points.

\begin{tabular}{|c|c|c|c|c|c|c|}
\hline Domain & Resolution level & $\Delta \mathrm{x} \times \Delta \mathrm{y}(\% / \mathrm{m})$ & $\Delta \mathrm{t}(\min )$ & $\mathrm{nf}$ & $\mathrm{n} \theta$ & $n g x \times n g y=n p$ \\
\hline \multirow{6}{*}{$\begin{array}{l}\text { West Iberian nearshore } \\
\text { Case study } 1\end{array}$} & Coarse (level I) & $0.05^{\circ} \times 0.1^{\circ}$ & 20 (non-stat) & 30 & 36 & $101 \times 101=10201$ \\
\hline & D1 North (level II) & $0.01^{\circ} \times 0.02^{\circ}$ & 20 (non-stat) & 30 & 36 & $101 \times 101=10201$ \\
\hline & D2 Center (level II) & $0.005^{\mathrm{o}} \times 0.005^{\mathrm{o}}$ & 20 (non-stat) & 30 & 36 & $101 \times 101=10201$ \\
\hline & D3 South (level II) & $0.02^{\circ} \times 0.02^{\circ}$ & 20 (non-stat) & 30 & 36 & $111 \times 76=8436$ \\
\hline & Local (level III) & $0.01^{\circ} \times 0.01^{\circ}$ & 1200 & 30 & 36 & $51 \times 51=2601$ \\
\hline & Cartesian HR (level IV) & $25 \mathrm{~m} \times 25 \mathrm{~m}$ & 3600 & 30 & 36 & $261 \times 201=52461$ \\
\hline \multirow{4}{*}{ Black Sea Case study 2} & Global (level I) & $0.08^{\circ} \times 0.08^{\circ}$ & 20 (non-stat) & 30 & 24 & $176 \times 76=13376$ \\
\hline & Coastal (level II) & $0.02^{\circ} \times 0.02^{\circ}$ & 20 (non-stat) & 30 & 36 & $141 \times 141=19881$ \\
\hline & Local (level III) & $0.005^{\circ} \times 0.005^{\circ}$ & $\begin{array}{c}20 \text { (non-stat) } \\
180 \text { (stat) }\end{array}$ & 30 & 36 & $161 \times 141=22701$ \\
\hline & Cartesian (level IV) & $50 \mathrm{~m} \times 50 \mathrm{~m}$ & 180 (stat) & 25 & 36 & $96 \times 107=10272$ \\
\hline \multirow{3}{*}{$\begin{array}{c}\text { Portuguese nearshore } \\
\text { Case study } 3\end{array}$} & Coastal (level I) & $350 \mathrm{~m} \times 300 \mathrm{~m}$ & 30 (stat) & 30 & 36 & $101 \times 101=10201$ \\
\hline & Local (level II) & $100 \mathrm{~m} \times 200 \mathrm{~m}$ & 30 (stat) & 30 & 36 & $121 \times 101=12221$ \\
\hline & $\begin{array}{l}\text { SHORECIRC } \\
\text { HR (level III) }\end{array}$ & $5 \mathrm{~m} \times 10 \mathrm{~m}$ rotation $\theta=-22^{\circ}$ & 30 (stat) & 1 & 1 & $181 \times 141=25521$ \\
\hline \multirow{2}{*}{$\begin{array}{c}\text { Sardinia island Case } \\
\text { study } 4\end{array}$} & Local (level I) & $25 \mathrm{~m} \times 25 \mathrm{~m}$ rotation $\theta=11^{\circ}$ & 30 (stat) & 35 & 60 & $135 \times 132=17820$ \\
\hline & $\begin{array}{c}\text { SHORECIRC } \\
\text { HR (level II) }\end{array}$ & $3 \mathrm{~m} \times 5 \mathrm{~m}$ rotation $\theta=11^{\circ}$ & 30 (stat) & 1 & 1 & $196 \times 111=21756$ \\
\hline \multirow{5}{*}{$\begin{array}{l}\text { Madeira archipelago } \\
\text { Case study } 5\end{array}$} & Large (level I) & $0.02^{\circ} \times 0.02^{\circ}$ & 20 (non-stat) & 30 & 36 & $51 \times 76=3876$ \\
\hline & Madeira MR (level II) & $700 \mathrm{~m} \times 700 \mathrm{~m}$ & 20 (non-stat) & 30 & 36 & $149 \times 109=16241$ \\
\hline & P. Santo MR (level II) & $200 \mathrm{~m} \times 200 \mathrm{~m}$ & 20 (non-stat) & 30 & 36 & $176 \times 169=29744$ \\
\hline & Madeira HR (level III) & $\begin{array}{c}300 \mathrm{~m} \times 100 \mathrm{~m} \\
\text { rotation } \theta=330^{\circ}\end{array}$ & $\begin{array}{c}20 \text { (non-stat) } \\
180 \text { (stat) } \\
\end{array}$ & 30 & 36 & $121 \times 151=17061$ \\
\hline & P. Santo HR (level III) & $\begin{array}{l}100 \mathrm{~m} \times 50 \mathrm{~m} \\
\text { rotation } \theta=45^{\circ}\end{array}$ & $\begin{array}{c}20 \text { (non-stat) } \\
180 \text { (stat) }\end{array}$ & 30 & 36 & $91 \times 141=12831$ \\
\hline \multirow{3}{*}{$\begin{array}{l}\text { Prestige accident Case } \\
\text { study } 6\end{array}$} & Coarse (level I) & $0.05^{\mathrm{o}} \times 0.05^{\mathrm{o}}$ & $\begin{array}{c}20 \text { (non-stat) } \\
360 \text { (stat) }\end{array}$ & 30 & 36 & $141 \times 121=17061$ \\
\hline & North (level II) & $0.02^{\circ} \times 0.01^{\mathrm{o}}$ & $\begin{array}{c}20 \text { (non-stat) } \\
360 \text { (stat) }\end{array}$ & 30 & 36 & $101 \times 101=10201$ \\
\hline & West (level II) & $0.01^{\circ} \times 0.02^{\circ}$ & $\begin{array}{c}20 \text { (non-stat) } \\
360 \text { (stat) }\end{array}$ & 30 & 36 & $101 \times 141=14241$ \\
\hline
\end{tabular}

(Faro, 7.9W, 36.9N). In order to illustrate the correlation between the significant wave heights measured in various points of the west Iberian coastal environment, time variations for the significant wave heights registered at the four buoys are presented in the upper side of Fig. 1 (day 1-1994//01/20- day 401994/02/28).

When implementing the SWAN model in a specific site, the simplest way would be to use the default mode that activates the parameterizations considered in general as most accurate and effective from computational point of view.

This is because the multitude of options existent in SWAN renders the model sometime confusing although at the same time makes it very flexible. Nevertheless, this default mode, though very often gives acceptable results, does not always use the entire potential of the model and better results can be obtained by tuning some parameters or activating alternative formulations for key physical processes. That is why some calibration studies to balance the accuracy of the predictions in the Iberian nearshore with the computational effectiveness, have been carried out and they will be briefly presented in this section. These studies were focused on the performances of the formulations available in SWAN to account for whitecapping dissipation. This is mainly because whitecapping is still considered the weak link in deep water wave modelling and it is closely related also with the process of wave generation.

Four different formulations are available to account for the 
Table 2. Case study 1. Wave statistics, the parameterizations Janssen-Hasselmann and Yan-A\&B, respectively were used for wave generation and whitecapping dissipation (simulations for the time interval 1994//01/20-1994/02/ 28). $B_{\text {med }}$ represents the average value of the measured wave parameters and $S_{\text {med }}$ the corresponding average value for the period considered of the wave parameters resulting from model simulations.

\begin{tabular}{|c|c|c|c|c|c|c|c|c|}
\hline \multirow{13}{*}{ JANSSEN } & & $\mathrm{B}_{\text {med }}$ & $S_{\text {med }}$ & Bias & RMSE & SI & $\mathrm{r}$ & Buoy \\
\hline & Hs (m) & 3.510 & 3.674 & -0.164 & 0.629 & 0.179 & 0.896 & \multirow{3}{*}{$\mathrm{B} 1(\mathrm{n}=242)$} \\
\hline & $\operatorname{Tm}(\mathrm{s})$ & 8.679 & 10.136 & -1.457 & 2.113 & 0.244 & 0.691 & \\
\hline & $\operatorname{Dir}\left(^{\circ}\right)$ & 298.49 & 289.01 & 9.477 & 15.456 & 0.052 & 0.798 & \\
\hline & Hs (m) & 3.250 & 3.524 & -0.274 & 0.604 & 0.186 & 0.906 & \multirow{3}{*}{$\mathrm{B} 2(\mathrm{n}=306)$} \\
\hline & $\operatorname{Tm}(\mathrm{s})$ & 8.491 & 10.270 & -1.780 & 2.355 & 0.277 & 0.656 & \\
\hline & $\operatorname{Dir}\left(^{\circ}\right)$ & 295.46 & 295.55 & -0.083 & 9.017 & 0.031 & 0.894 & \\
\hline & Hs (m) & 2.522 & 2.804 & -0.282 & 0.456 & 0.181 & 0.963 & \multirow{3}{*}{$\mathrm{B} 3(\mathrm{n}=124)$} \\
\hline & $\operatorname{Tm}(\mathrm{s})$ & 9.110 & 10.495 & -1.386 & 1.982 & 0.218 & 0.470 & \\
\hline & $\operatorname{Dir}\left(^{\circ}\right)$ & 306.27 & 299.48 & 6.796 & 9.355 & 0.031 & 0.522 & \\
\hline & Hs (m) & 1.207 & 1.169 & 0.038 & 0.292 & 0.242 & 0.898 & \multirow{3}{*}{$\mathrm{B} 4(\mathrm{n}=320)$} \\
\hline & $\operatorname{Tm}(\mathrm{s})$ & 5.703 & 5.049 & 0.654 & 1.211 & 0.212 & 0.808 & \\
\hline & $\operatorname{Dir}\left(^{\circ}\right)$ & 223.77 & 215.12 & 8.649 & 33.523 & 0.150 & 0.747 & \\
\hline \multirow{12}{*}{$A \& B$} & $\mathrm{Hs}(\mathrm{m})$ & 3.510 & 3.082 & 0.427 & 0.745 & 0.212 & 0.895 & \multirow{3}{*}{$\mathrm{B} 1(\mathrm{n}=242)$} \\
\hline & $\operatorname{Tm}(\mathrm{s})$ & 8.679 & 0.024 & -0.345 & 2.177 & 0.251 & 0.613 & \\
\hline & $\operatorname{Dir}\left(^{\circ}\right)$ & 298.49 & 287.404 & 11.087 & 17.641 & 0.059 & 0.765 & \\
\hline & Hs (m) & 3.250 & 2.924 & 0.325 & 0.622 & 0.192 & 0.908 & \multirow{3}{*}{$\mathrm{B} 2(\mathrm{n}=306)$} \\
\hline & $\operatorname{Tm}(\mathrm{s})$ & 8.491 & 9.281 & -0.790 & 2.271 & 0.267 & 0.591 & \\
\hline & $\operatorname{Dir}\left(^{\circ}\right)$ & 295.46 & 293.81 & 1.657 & 9.641 & 0.033 & 0.884 & \\
\hline & Hs (m) & 2.522 & 2.272 & 0.250 & 0.408 & 0.162 & 0.970 & \multirow{3}{*}{ B3 $(n=124)$} \\
\hline & $\operatorname{Tm}(\mathrm{s})$ & 9.110 & 9.494 & -0.385 & 2.666 & 0.293 & 0.194 & \\
\hline & $\operatorname{Dir}\left(^{\circ}\right)$ & 306.27 & 299.21 & 7.065 & 9.890 & 0.032 & 0.438 & \\
\hline & Hs (m) & 1.207 & 0.922 & 0.285 & 0.470 & 0.390 & 0.832 & \multirow{3}{*}{$\mathrm{B} 4(\mathrm{n}=320)$} \\
\hline & $\operatorname{Tm}(\mathrm{s})$ & 5.703 & 4.194 & 1.509 & 2.452 & 0.430 & 0.555 & \\
\hline & $\operatorname{Dir}\left(^{\circ}\right)$ & 223.77 & 200.15 & 23.620 & 45.324 & 0.203 & 0.550 & \\
\hline
\end{tabular}

processes of wave generation by wind and whitecapping dissipation. These are: a) Komen's parameterization [15], to describe the transfer of wind energy to the waves coupled with the pulse-based model of [10], for whitecapping dissipation, as adapted by the WAMDI group [33] to be applicable in finite water depth, b) Janssen's model for atmospheric input [13], coupled with the same pulse-based model of Hasselmann [15] for whitecapping, c) Komen's model coupled with the $\mathrm{Cu}$ mulative Steepness Method [32] for whitecapping, d) Yan's model for atmospheric input [34] coupled with the saturation based model of Alves and Banner [1], for whitecapping (that will be denoted in this work as A\&B).

The first parameterization (Komen - Hasselmann) corresponds also to WAM cycle 3 while the second one (Janssen Hasselmann) corresponds to WAM cycle 4. CSM is an alternative formulation for whitecapping and with this method dissipation due to whitecapping depends on the steepness of the wave spectrum at and below a particular frequency. A second alternative for the whitecapping expression uses the formulation of Alves and Banner, [1]. This expression is based on experimental findings that whitecapping dissipation appears to be related to the nonlinear hydrodynamics within wave groups. This yields a dissipation term that primarily depends on quantities that are local in the frequency spectrum, opposed to ones that are distributed over the spectrum, as in the expression of Komen [15]. This whitecapping expression is used together with a wind input term that is based on Yan, [34].

Model system simulations were performed for a forty-day period at the beginning of 1994 when data from all four buoys were available (between 1994/01/20 and 1994/02/28). The wind field for all the simulations was provided by the project HIPOCAS "Hindcast of Dynamic Processes of the Ocean and Coastal Areas of Europe," developed in the framework of the European Program "Energy, Environment and Sustainable Development" [9], which gave the reanalysis wind conditions for 44 years, between 1958 and 2001.

All four options discussed above for atmospheric input and whitecapping dissipation were considered separately in order to test the system reliability. Table 2 presents the results for the two whitecapping parameterisations that perform better. Es- 
timations of statistical parameters were carried out for all the four buoys. Usually the error statistics in wave modeling assumes the estimation of some parameters like: mean values, bias, RMSE (root-mean-square-error), SI (scatter index) and $\mathrm{r}$ (correlation coefficient also called Pearson's product momentum correlation). If $X_{i}$ represents the measured values, $Y_{i}$ the simulated values and $n$ the number of observations the above mentioned parameters can be defined with the relationships:

$$
\begin{gathered}
X_{\text {med }}=\tilde{X}=\frac{\sum_{i=1}^{n} X_{i}}{n} \quad \text { Bias }=\frac{\sum_{i=1}^{n}\left(X_{i}-Y_{i}\right)}{n} \\
R M S E=\sqrt{\frac{\sum_{i=1}^{n}\left(X_{i}-Y_{i}\right)^{2}}{n}} \\
S I=\frac{R M S E}{\tilde{X}} r=\frac{\sum_{i=1}^{n}\left(X_{i}-\tilde{X}\right)\left(Y_{i}-\tilde{Y}\right)}{\left(\sum_{i=1}^{n}\left(X_{i}-\tilde{X}\right)^{2} \sum_{i=1}^{n}\left(Y_{i}-\tilde{Y}\right)^{2}\right)^{\frac{1}{2}}}
\end{gathered}
$$

Some observations concerning the results provided by the wave prediction system in the Iberian nearshore can be made at this point:

a) In general, in terms of bias, RMSE and SI, the results are better when using the parameterization Janssen-Hasselmann for wave generation and whitecapping dissipation, respectively. On the second place is the new formulation Yan-A\&B.

b) In terms of correlation coefficients, there are no fundamental differences between all the four formulations. However, as regards the significant wave heights the results are slightly better when using the Komen-Hasselmann formulation, while for the wave directions Janssen-Hasselmann still gives slightly better results. Lower correlations can be noticed in general in relationship with the wave periods.

c) At Buoy 3, although the significant wave height is better correlated, the lowest correlation in relationship with the wave direction is encountered.

Hence, when modelling waves close to the west Iberian coastal environment, Janssen-Hasselmann formulation for wind input and whitecapping dissipation appears to be the most effective. Moreover, this is also consistent with the generation module since WAM cycle 4 uses exactly the same formulation. The new introduced model Yan-A\&B looks very promising and is in general considered most appropriate for mixed swell wind seas conditions.

The results presented and analyzed in this section show that the most reliable scheme for wave modelling in the west Iberian coastal environment would be a flexible one that uses in

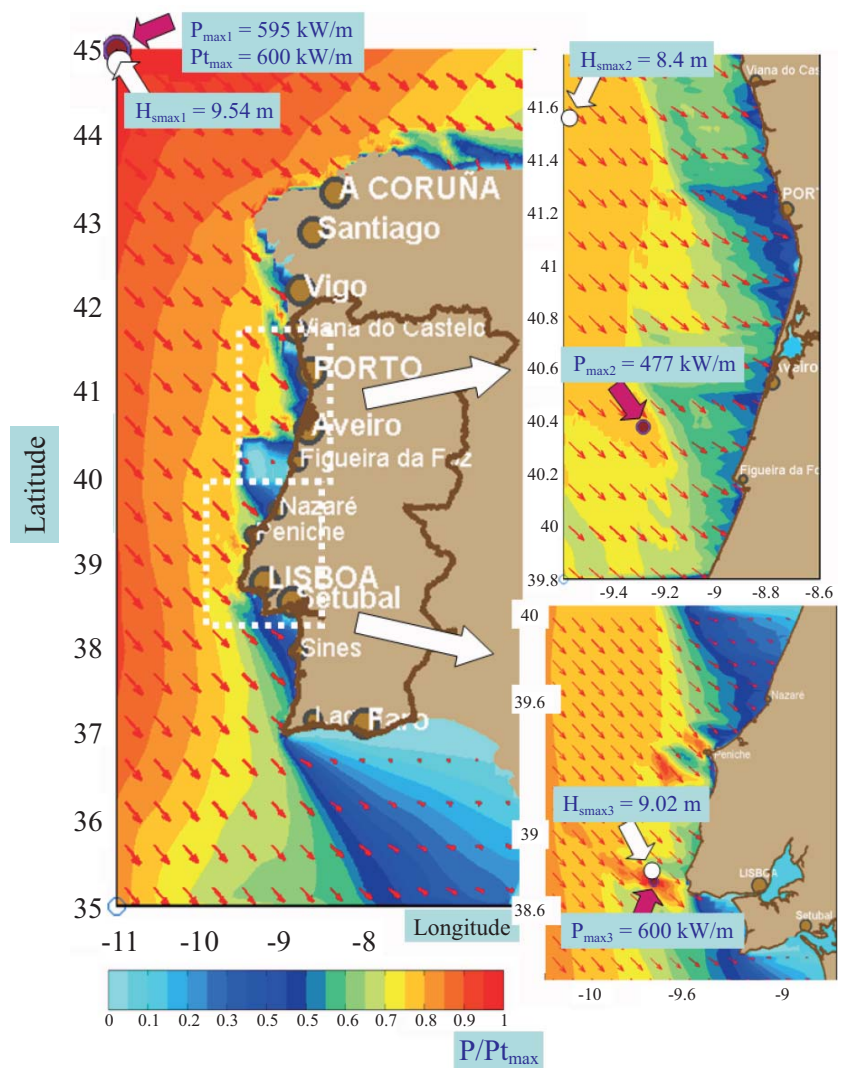

Fig. 2. Case study 1. Results from the West Iberian wave driver and two medium resolution computational domains; in background normalized wave power, in foreground energy transport vectors (in $\mathrm{kW} / \mathrm{m}$ of wave front). Time frame 1994/02/04_h15, energetic peak.

general the Janssen-Hasselmann parameterization for wind input and whitecapping dissipation and replaces it with the formulation Yan-A\&B in the cases when the wind sea component becomes relevant.

Once the calibration and some validation tests have been performed, the wave prediction system can be focused on various coastal areas as in the example illustrated in Fig. 2 when the target area is the Portuguese port of Sines and the time frame considered is a high energetic situation (1994/02/ 04/h15).

For the same time frame, Fig. 3 illustrates the spatial distribution of the wave energy. The west Iberian nearshore has historically proved as a very popular location for wave energy developers with some companies considering testing prototype devices here. Hence the wave energy assessments are of special interest in this particular coastal environment.

In SWAN, the energy transport components (expressed in $\mathrm{W} / \mathrm{m}$, i.e. energy transport per unit length of wave front), are computed with the relationships:

$$
\begin{aligned}
& P_{x}=\rho g \iint C_{g x} E(\sigma, \theta) d \sigma d \theta \\
& P_{y}=\rho g \iint C_{g y} E(\sigma, \theta) d \sigma d \theta,
\end{aligned}
$$






Fig. 3. Case study 1. Model system focalization on the Portuguese port of Sines, time frame 1994/02/04/h15. In background the significant wave height scalar fields are represented while in foreground (with black arrows) the wave direction.

where $x, y$ are the problem coordinate system (for the spherical coordinates $x$ axis corresponds to longitude and $y$ axis to latitude), and $C_{g x}, C_{g y}$ are the components of the group velocity defined in Eq. (5). Hence the absolute value of the energy transport (denoted also as wave power) will be:

$$
P=\sqrt{P_{x}^{2}+P_{y}^{2}}
$$

The horizontal analysis illustrated in Fig. 3 was performed considering first the regional computational level covering the entire western Iberian coast. In a second approach, two medium resolution domains were also defined in order to cover both the northern and central Portuguese coastal environments. The first medium resolution domain corresponds with the computational domain formerly denoted as D1 (illustrated in Fig. 1), whereas the second medium resolution domain has been additionally introduced to model the wave transformation in the central part of the Portuguese nearshore. Its resolutions in geographical and spectral spaces are identical with those defined for the foregoing domain.

The non dimensional normalized wave power was expressed as:

$$
P_{n}=\frac{P}{P_{t \max }} .
$$

For this particular situation the value $P_{t \max }$ was set to 600

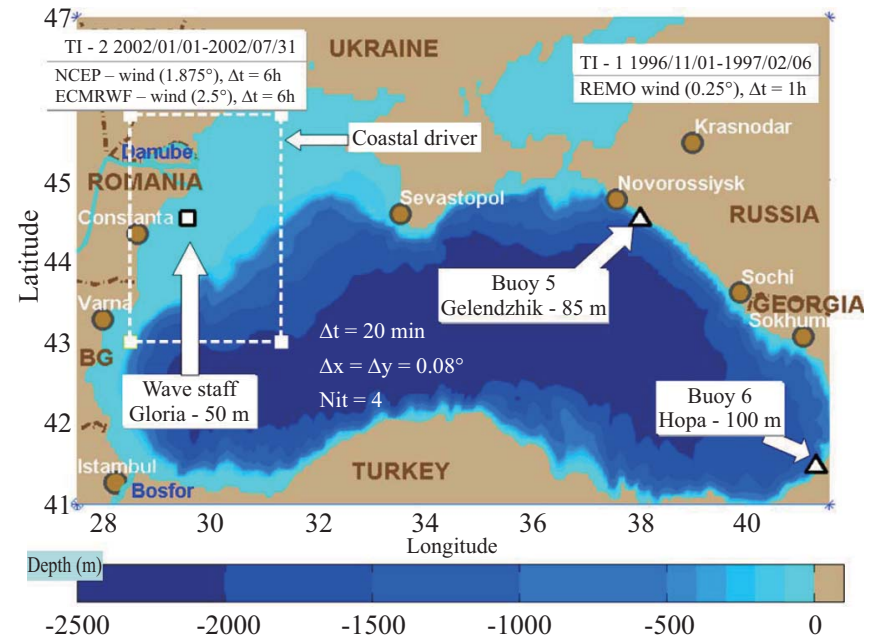

Fig. 4. Case study 2. The bathymetric map of the Black Sea basin corresponding to the computational domain and the locations of the three data sources available. The two time intervals considered for the wave hindcast are also indicated in the figure.

$\mathrm{kW} / \mathrm{m}$. The case presented reflects a normal energetic peak for wintertime and not an extreme event. Fig. 3 presents the normalized wave power fields in background, and the energy transport vectors in foreground.

The maximum values for wave power and significant wave height in each computational domain are also indicated in Fig. 3 while the corresponding locations are marked with circles.

Some observations arise from the analysis of the above results. Although there is an obvious relation between the significant wave height and the wave power, the energetic peak in a computational domain is not necessarily located in the same point (or even close) with the maximum significant wave height. This is very well illustrated by the results presented in Fig. 3 related to the northern medium resolution simulations. A second important observation would be that the local effects may induce sometime energetic peaks that exceed the global peak from the coarse area (the West Iberian driver), and this is illustrated in Fig. 3 for the medium resolution simulation corresponding to the central area where an energetic peak is greater than $600 \mathrm{~kW} / \mathrm{h}$ occurred. Further details on the wave energy assessments in the west Iberian nearshore are given in [22].

\section{Case study 2 - Wave Hindcast in the Black Sea}

A wave predictions system entirely based on the SWAN model was implemented and tested in the Black Sea basin. This approach has the advantage that the same wave model covers the full scale of the modeling process, although the physical model parameterizations are rather different from one computational level to another.

The computational domain considered for the present SWAN implementation is illustrated in Fig. 4. The system origin corresponds to the lower left corner point and has the coordinates $\left(27.5^{\circ}, 41.0^{\circ}\right)$. The $x$-direction (longitude) ex- 
tends $14^{\circ}$ and the $y$-direction (latitude) extends $6^{\circ}$, covering both the Black Sea and the Sea of Azov.

Three check points were considered for model calibration and for performing validation tests. Data coming from two directional buoys operating in deep water, close to the east coast, were first considered. As a second step, the reliability of the SWAN model results was tested on the west coast using two different wind fields. Results were compared with measurements provided by a wave staff located at the Gloria drilling platform that is operating in the area (illustrated also in Fig. 4).

These studies were focused on how well the formulations available in SWAN were able to account for whitecapping dissipation. As indicated in Fig. 4, two time intervals were considered for model system calibration and for performing some validation tests. The first phase corresponds to the time interval between 1 November 1996 and 6 February 1997, denoted as TI-1. For this interval, model results were compared with data from two buoys operating on the east coast of the sea. These are Gelendzihik (37.98E, 44.51N), denoted as B5, and Hopa (41.38E, 41.42N), denoted as B6. They are both located in deep water, at 85 and 100 meters depth, respectively.

The wind field for this period was provided by the HIPOCAS project [9]. During this first phase, for wave simulations for the time interval TI-1, the global NCEP reanalysis wind was used as a driver for the regional atmosphere model REMO. The spatial resolution of the wind model output was $0.25^{\circ}$ and the time step was one hour.

Simulations were performed employing most of the possibilities for tuning the SWAN model to account for deep water wave processes. As mentioned previously, four options are currently available in SWAN to account for whitecapping dissipation and wind generation of waves.

Analysis of the statistical data [20] shows that, for most of the parameters studied (i.e., significant wave height, mean period, peak period and mean wave direction), best agreement with data measured at B5 occurs using the A\&B formulation followed closely by Komen's formulation. The results also indicate that the A\&B formulation not only yields the best predictions, but also requires the smallest variation of the tuneable coefficients. Direct comparisons SWAN against B5 for the main wave parameters $(\mathrm{Hs}, \mathrm{Tm}$ and mean wave direction), are presented in Fig. 5. The results provided by the parameterizations Komen-Hasselmann and Yan-A\&B that gave better results are illustrated in parallel.

The performance of the model was also assessed in the southern portion of the east coast for the same time interval (TI-1). The Hopa directional buoy (B6) was operating in that area and its location is indicated in Fig. 4.

This time only the Komen and A\&B whitecapping formulations were evaluated, since they were found to be more effective in the previous case. Significant wave height results provided by the A\&B formulation are still better than those obtained with the Komen-Hasselmann approach. The Komen formulation gives slightly better results for the mean period.



Fig. 5. Case study 2. Direct comparisons SWAN against B5 (Hs, Tm and mean wave direction), day 1-1996//11/01- day 98-1996/02/06, (TI-1). The results provided by the parameterizations KomenHasselmann and Yan-A\&B to model wave generation and whitecapping dissipations were illustrated in parallel.

Although the model does not provide very accurate predictions of the mean directions, the A\&B formulation performs slightly better. When compared with the results for B5 it can be seen that significant wave heights are better estimated at B6 in terms of RMS and at B5 in terms of scatter indices. The mean periods and mean directions are better estimated at B5.

As a second validation test, model simulations were performed for the time interval between 1 January 2002 and 31 July 2002 (denoted as TI-2). Two different wind fields were used: NCEP $\left(1.875^{\circ}\right.$ spatial resolution) and ECMWF $\left(2.5^{\circ}\right.$ spatial resolution); the temporal resolution is 6 hours for both fields. Data measured in situ by a wave staff placed at the Gloria drilling platform were used as a reference. The drilling unit operates in the western coastal environment of the Black Sea at about $50 \mathrm{~m}$ water depth (Fig. 4), and both wind and wave measurements were available for the period considered. Since the wave staff was located in intermediate water depth, a second area was connected to the main computational domain in order better to account for the coastal wave transformation, as illustrated in Fig. 4. Comparisons indicate that the wind model predictions were generally reliable. The NCEP wind fields appear to be more accurate; however, the atmospheric models seem to systematically under predict the wind velocities (positive biases)

Using the KOMEN and A\&B formulations, SWAN simulations were performed considering the NCEP and ECMWF wind fields. As expected, NCEP field provided better results in terms of the wave parameters than ECMWF. The results are again slightly better when the A\&B formulation is used. 




Fig. 6. Case study 2. Nearshore focusing towards the western coast, significant wave height fields and wave vectors, case study corresponding to the time frame $2002 / 03 / 11 / h 18$, typical storm.

Nevertheless, the results are, in general, less accurate than for the case discussed in the previous section when the wind resolution was better both in space and time.

At high energetic conditions, significant wave height peaks seem to be systematically underestimated by the model when the $\mathrm{A} \& \mathrm{~B}$ formulation is used while the Komen formulation appears to be more effective in this high energetic range. To account for this and improve the performance of the model predictions, the A\&B formulation could be employed for low and moderate energetic conditions, and the Komen formulation should be applied for high energetic conditions. In order to achieve this, a dynamic modeling scheme was designed to switch from one witecapping model to another. The driving parameters are the maximum values of the wind velocity and the significant wave height. Thus if the maximum value of wind velocity is greater than $20 \mathrm{~m} / \mathrm{s}$ or the maximum value of the significant wave height (imposed as input on the boundaries) is greater than 6 meters the simulation is performed using Komen formulation while A\&B parameterization is activated otherwise.

The model system focalization for the most energetic situation from the first time interval considered is illustrated in Fig. 6 . The time frame presented is $2002 / 03 / 11 / \mathrm{h} 18$, and represents a typical storm while the target area is the Romanian port of
Mangalia. The characteristics of the computational domains considered are presented in Table 1.

\section{NOWCAST SCHEMES}

\section{Case study 3 - Nowcast in the Portuguese Nearshore}

The next two case studies that will be discussed are nowcast schemes, one in the central part of the Portuguese nearshore and the other in the coastal environment of Sardinia Island in the Mediterranean Sea.

The third case study considered, illustrates a nowcast scheme for waves and nearshore currents in a coastal sector located in the central part of the Portuguese nearshore. It is a 9-km long uniform coastal sector with a straight shoreline parallel to the depth contours approximately oriented $\mathrm{N} 21^{\circ} \mathrm{E}$. It is marked by the absence of coastal structures, either natural (like rocky outcrops) or artificial (normal to the shoreline, like groins, or parallel, like breakwaters), and free of discontinuities (like river mouths). It is a sandy ocean bottom zone without sediment sources or sinks. The site bathymetry is illustrated in Fig. 7(a) (in the upper side) interpolated from a 1994 hydrographical chart of scale 1:2,000. A continuous submarine bar rises above the $10 \mathrm{~m}$ depth contour, reaching minimum soundings of $4 \mathrm{~m}$. The bar dimensions are 3.5-6 m height and $350-450 \mathrm{~m}$ width. The bar crest is $500-650 \mathrm{~m}$ from the mean sea level coastline.

In order to provide a realistic estimate of the nearshore waves and littoral drift current, and to contribute to the numerical models calibration in the target area, a field experiment was performed [27] by releasing and tracking, through geo-referenced sequenced aerial photographs, of a substantial number of surface drifters at two previously selected points just off the surf zone.

Sea and swell conditions approaching the nearshore area, as well as tide and background current over the inner shelf, were monitored at the outer limit of the experiment area with an Acoustic Doppler Current Profiler (ADCP) equipped with a wave module moored at the 13-m depth contour (Fig. 7(a)). The predominant waves in this region are from NW, with significant wave heights commonly between 1 and $2 \mathrm{~m}$, and mean periods between 5 and $7 \mathrm{~s}$. The wind conditions were observed in a coastal station $60 \mathrm{~km}$ south of the experiment area.

The field experiment concluded with 120 photographs that were scanned and geo-referenced with known points in land. The central coordinates of the photographs were compared with those provided by the in-flight navigation system, giving maximum differences of $10 \mathrm{~m}$ in $\mathrm{N}-\mathrm{S}$ direction and $2 \mathrm{~m}$ in $\mathrm{E}-\mathrm{W}$ direction. Envelopes of markers groups were drawn, and vector positioning of individual markers was used for determining the group mass centre position [7]. Approximate speed estimates were obtained from the shortest distance between consecutive centre positions.

For the observed wave conditions corresponding to typical values at that site, the significant longshore current was greater 


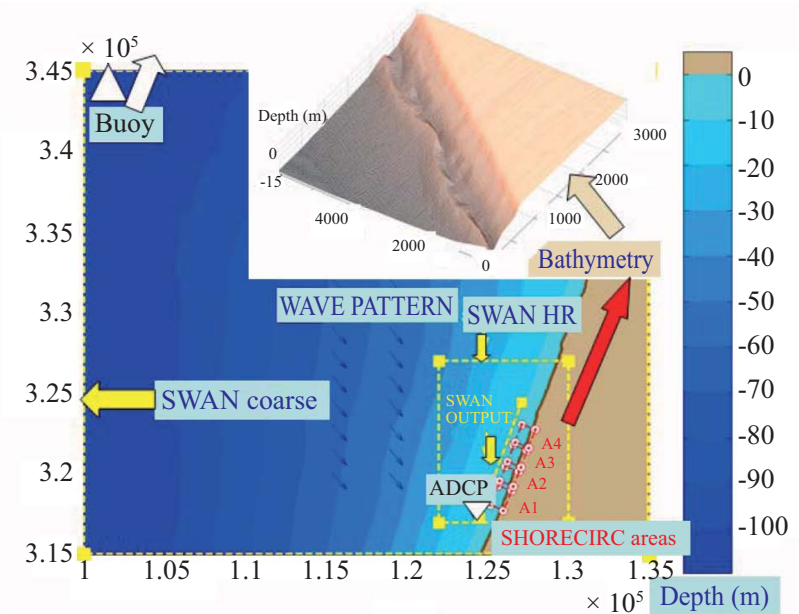

(a)

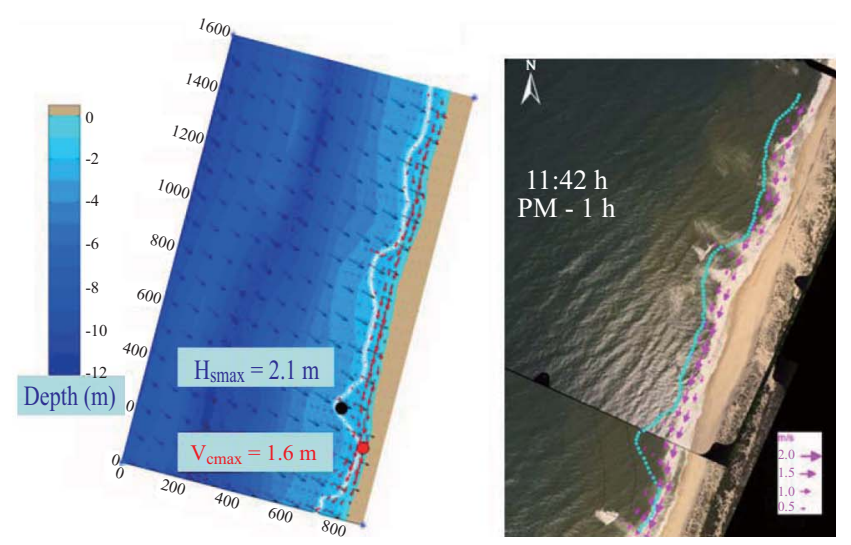

(b)

Fig. 7. Case study 3. (a) The computational domains, location of the data sources and the 3D representation of the high resolution bathymetry; (b) Left side-SHORECIRC results for the second computational domain (A2), time frame 2003/10/07/h11.42 high tide situation. In foreground, the wave vectors (black arrows), nearshore current vectors (red arrows), and breaking line are represented, while in background the 2D bathymetric map is illustrated; (b) Right side - nearshore currents resulting from SHORECIRC simulations superposed on the photography of the target area (A2) for the same time frame considered before (2003/10/07/h11.42).

than $20 \mathrm{~cm} / \mathrm{s}$ up to $1 \mathrm{~m} / \mathrm{s}$, for the markers caught within the surf zone, with $30 \mathrm{~cm} / \mathrm{s}$ average and $21 \mathrm{~cm} / \mathrm{s}$ standard deviation. The markers that drifted seawards had a lower speed, of the same order of magnitude of the surface current as measured by the ADCP. The speed of cross-shore drift was less than $15 \mathrm{~cm} / \mathrm{s}$ in consistency with the cross-shore surface current observed by the current profiler.

The computational strategy adopted for this case study is illustrated in Fig. 7(a). The main data source is a wave rider type directional buoy located at about 90 meters water depth in the North West of the target area. The wave transformation is simulated using two successive SWAN computational domains with increasing resolution shoreward. The calculated wave parameters were compared to the ADCP data. The
SWAN output along a line defined almost parallel to the coast (Fig. 7(a)) was input in the two circulation models.

Four small computational domains covering the target area were used with SHORECIRC [30]. This is a quasi-3D model that combines a numerical solution for the depth-integrated $2 \mathrm{D}$ horizontal momentum balance equations with an analytical solution for the $3 \mathrm{D}$ current profiles. Because of the greater number of the grid points, the computational time required for one grid for the entire domain was too large. The four SHORECIRC computational domains permitted concurrent computations. The characteristics of the computational domains considered are presented in Table 1.

Simulations were performed for the conditions that occurred on 7 October 2003, for 2 hours and 45 minutes (between 9am and $11.45 \mathrm{am}$ ) every 15 minutes. At each time, the input conditions and the tide level were varied.

An example of visual validation concerning the breaking characteristics is illustrated in Fig. 7(b). In the left side of this figure, SHORECIRC results for the second computational domain A2 are presented for 2003/10/07/h11.42 at high tide situation. Wave vectors (black arrows), nearshore current vectors (red arrows), and breaking line are shown in foreground and 2D bathymetry in background. On the right side, nearshore currents and breaking line resulting from SHORECIRC simulations are shown. A good agreement of the breaking characteristics is obtained.

The current velocities from simulations are in the same range as field data. The breaking ratio considered in simulations is:

$$
\gamma=\frac{H_{r m s}}{h_{0}}=0.5
$$

with the root-mean-square wave height $\left(H_{r m s}\right)$ derived from the significant wave height considering the Rayleigh distribution; i.e. $H_{r m s}=0.707 H_{s}$. The value of 0.5 for this parameter produced the best comparison with the corresponding photographs. The effect of the wind stress was also accounted by including for each time step the values of wind velocity and direction in the SHORECIRC input file. In this way the model will include the wind contribution in the nearshore current. The wind input for this case was provided by the meteorological station of the National Portuguese Institute of Meteorology located close to the target area.

The time step $(\Delta t)$ in SHORECIRC is controlled by the Courant number, defined as

$$
C r=\sqrt{g h_{m}} \Delta t / \Delta x
$$

where $h_{m}$ represents the maximum water depth in the computational domain, $\Delta x$ the spatial step in the cross-shore direction, and $g$ the acceleration of gravity. The Courant number was 0.1 , and time step was 0.045 seconds. To reach a steady state flow, at least 10,000 time steps were required. 


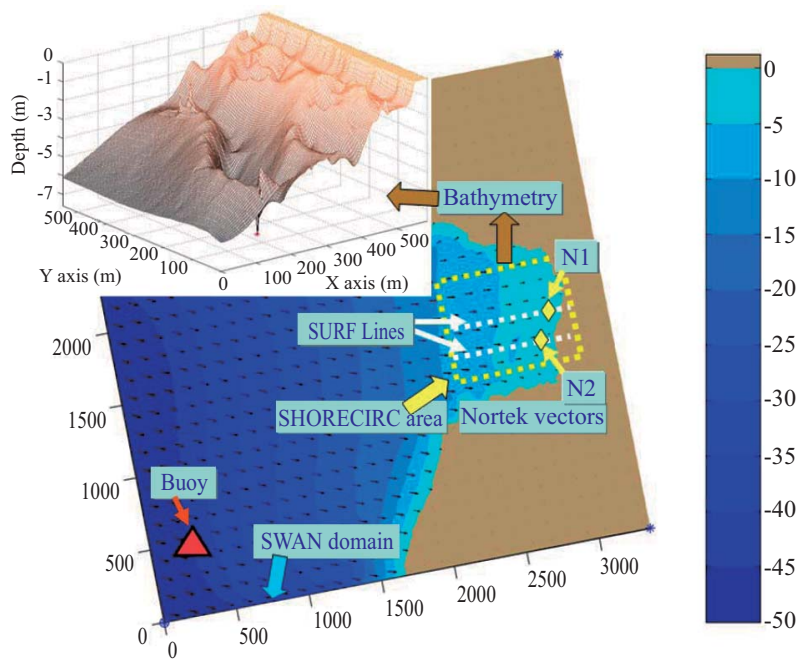

Fig. 8. Case study 4. Porto Ferro, Sardinia - nonconventional coastal environment with strong bathymetric irregularities; the computational domains, location of the data sources and the $3 \mathrm{D}$ representation of the high resolution bathymetry.

In general, a good agreement between the model results and the measurements was encountered. Further details concerning the effectiveness of this nowcast scheme are given in [23].

\section{Case study 4 -A Nowcast Scheme in Sardinia}

The fourth case study considered illustrates a nowcast scheme in Porto Ferro, located on the northwest coast of Sardinia near the city of Alghero. Porto Ferro is a westward opening bay and it is fully open to the strongest winter weather conditions in the western Mediterranean generated by the Mistral wind blowing out of the Gulf of Lion.

A high quality field site bathymetry was obtained by combining data from several surveying techniques. The shoreline and the beach topography were surveyed by foot and by landbased vehicle with a GPS receiver. An echo-sounder and a GPS receiver mounted on a work boat surveyed the shallow water region $(2-10 \mathrm{~m})$, whereas a vessel based multibeam system surveyed deeper waters $(12-50 \mathrm{~m})$. The echo-sounder survey was performed with $50 \mathrm{~m}$ resolution in the longshore with approximately $1 \mathrm{~m}$ resolution in the cross-shore, while the final multibeam valid data had spatial resolution of $1 \mathrm{~m}$ or higher. All these data were merged and bottom depth values were referred to local mean sea level. A $3 \mathrm{D}$ view of the bathymetry in the high resolution area is shown in Fig. 8. Although the mean beach slope can be considered as mild, the figure makes it clear that this location is representative of a site with strongly 3-D characteristics.

The computational scheme is suggested in Fig. 8. A waverider type buoy was considered as offshore forcing source for the SWAN model simulations. The 10-m depth isoline defines the external boundary of the two models (ISSM and SHORECIRC). ISSM, acronym for the Interface for SWAN and Surf Models [21], is an easily operable computational system that has been designed to simulate waves and longshore currents. The system is composed of a MATLAB GUI in the foreground, which directs the integration of the SWAN shallow water wave model with the 1D SURF model [17] in the background.

The characteristics of the computational domains for wave and current modeling are presented in Table 1. Two Nortek gauges (denoted as N1 and N2) provided the nearshore wave and current measurements. A detailed description of the field trial performed for wave and current data acquisition in February 2005, in Porto Ferro, is provided by [5].

In nowcast schemes, the comparison in variations is often more suggestive than direct comparison of the significant wave height $(H s)$. This is the comparison between $H s$ differences as resulting from the offshore-nearshore measurements $\left(\Delta H s_{\text {measured }}\right)$ against the differences between $H s$ resulted from SWAN simulations at the points where the instruments are located $\left(\Delta H s_{\text {simulated }}\right)$. Such comparison of the differences between $H s$ from the offshore-nearshore measurements against the differences between $H s$ resulted from SWAN simulations at the points where the offshore and nearshore instruments are located, is shown in Fig. 9(a) for a 9-day period starting on 2005/02/06/h12. The N1 Nortek vector was considered as reference nearshore data source. The scatter plot for the same parameter is presented in Fig. 9(b). Both figures show that SWAN predictions are reasonable for significant wave heights even for such complicate nearshore bathymetric conditions. Table 3 presents the statistical results corresponding to this nowcast scheme for the time period considered above.

Nevertheless for such complicated bathymetry SHORECIRC model had problems in 3D mode runs. For this reason, the simulations for the third case study were performed using the 2D mode of SHORECIRC. The 2D version of the model was enhanced by including an extra term in the modified radiation stress that enhances lateral mixing in comparison with the standard 2D model. With the 2D version of the SHORECIRC it is necessary to substantially increase the eddy viscosity to compensate for the missing dispersive mixing in order to obtain reasonable results.

\section{OPERATIONAL FORECAST}

\section{Case study 5 - Operational Forecast in Madeira Archipelago}

The last two case studies that will be discussed in this work present some forecast products provided by wave modeling systems in Madeira Archipelago for the case of the NATO exercise Unified Odyssey 2002 and in the case of the environmental alert generated by the accident of the oil carrier Prestige offshore the Iberian coasts in November 2002.

At the end of January and beginning of February 2002 the NATO exercise 'Unified Odyssey 2002' was held. This application was located in the archipelago of Madeira especially focused in the southern part of the Porto Santo Island where an 
Table 3. Case study 4. Nearshore wave statistics corresponding to the period 2005/02/06/h12-2005/02/14/h20.

\begin{tabular}{|c|c|c|c|c|c|c|}
\hline & Bias & RMSE & SI & r & Model & Vector-mean depth (m) \\
\hline \multicolumn{7}{|c|}{ Waves } \\
\hline Hs (m) & 0.075 & 0.095 & 0.286 & 0.823 & \multirow{2}{*}{ NWAN } & \multirow{2}{*}{ N1- 1.24 } \\
\hline Tp (s) & 0.049 & 0.642 & 0.164 & 0.785 & \multirow{2}{*}{ SWren } & \\
\hline Dir ( $\left.{ }^{\circ}\right)$ & -3.79 & 6.04 & 0.02 & 0.441 & \\
\hline \multicolumn{7}{|c|}{ Correlation offshore-nearshore in waves prediction } \\
\hline Hs (m) & -0.026 & 0.137 & 0.152 & 0.994 & SWAN & B1- 76 --N1- 1.24 \\
\hline
\end{tabular}

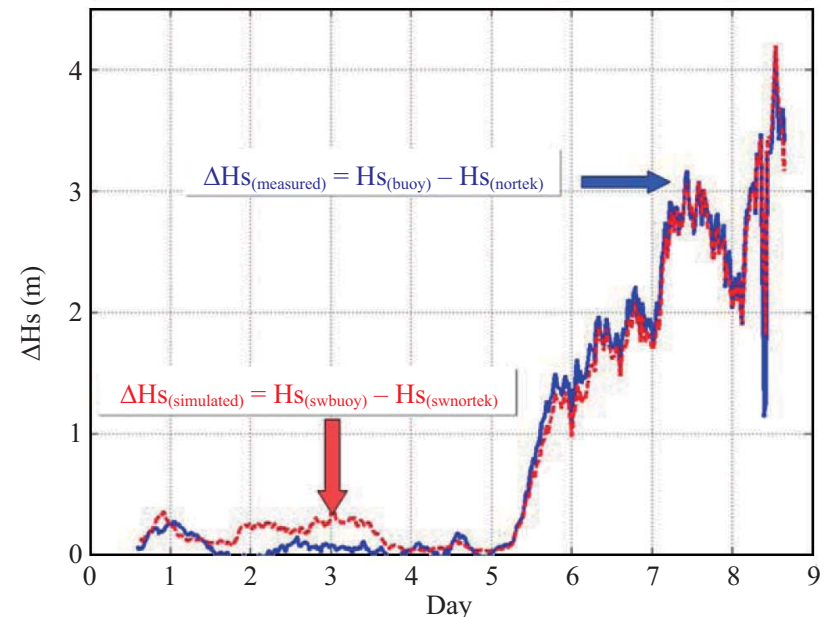

(a)

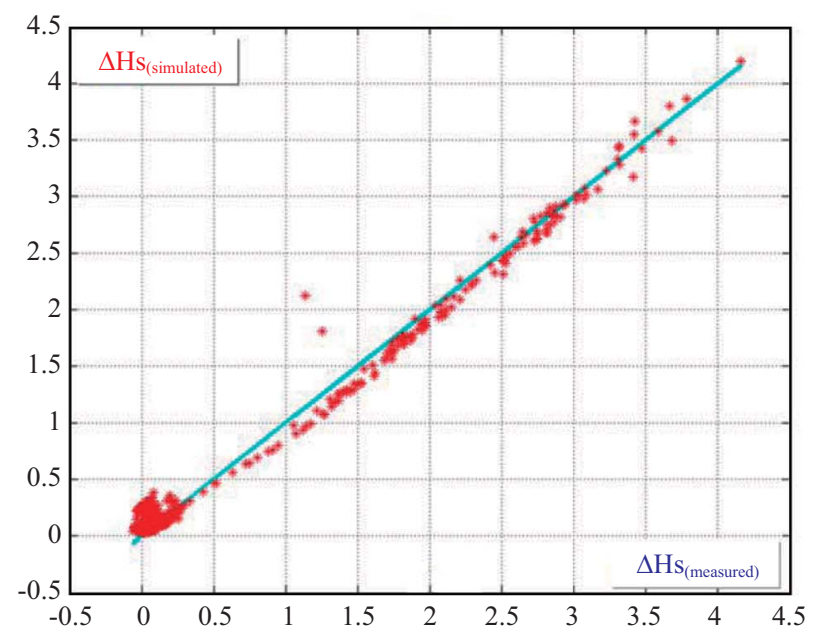

(b)

Fig. 9. Case study 4. (a) Comparisons of the computed (SWAN), and measured, offshore-nearshore significant wave height differences (day $1=2005 / 02 / 06)$. (b) Scatter plot for the offshore-nearshore significant wave height differences, simulations (SWAN) versus measurements ( 388 data points)

oil terminal was operating at that time. Some general features of the operational wave forecast performed with that occasion will be presented in this section. A detailed description of the modeling techniques adopted is given in [25].

The computational strategy is suggested in Fig. 10(a). The


(b)

Fig. 10. Case study 5. (a) Computational levels assumed for the wave forecast in Madeira Archipelago, SWAN coastal focusing and location of the data sources (B7 and B8). (b) Average wave conditions in Madeira Archipelago (2002/02/02 midday - 12 hours forecast) - significant wave height fields and wave vectors for Porto Santo medium resolution simulation (level 2)

wave forecast was based on simulations with SWAN. The initial forcing conditions, concerning the nowcast and threeday forecast with 12 hours time step, were provided by the 'Fleet Numerical Meteorology and Oceanography Center' (FNMOC). Thus the wave parameters came from the WW3 simulations while for the wind was considered the NOGAPS field data. The resolution of the WW3 simulations was of $0.92^{\circ}$ in the north direction and $1.25^{\circ}$ towards west. However, in the area of the Porto Santo Island this resolution was increased to $0.33^{\circ}$ towards north and respectively $0.42^{\circ}$ in the west direction. The SWAN simulations were performed both 
Table 4. Case study 5. Statistical results for the wave parameters (simulations for the time interval 07/10/1997-01/03/ 1998). B7 - Funchal, B8 - Caniçal.

\begin{tabular}{|c|c|c|c|c|c|c|c|c|}
\hline & & $\mathrm{B}_{\text {med }}$ & $S_{\text {med }}$ & Bias & RMSE & SI & $\mathrm{r}$ & Buoy \\
\hline \multirow{3}{*}{ LEV 1} & Hs (m) & 1.28 & 1.36 & -0.08 & 0.35 & 0.27 & 0.89 & \multirow{9}{*}{$\begin{array}{l}\text { B7 Funchal } \\
(\mathrm{n}=1067)\end{array}$} \\
\hline & $\operatorname{Tm}(\mathrm{s})$ & 5.67 & 5.38 & 0.29 & 1.46 & 0.26 & 0.58 & \\
\hline & $\operatorname{Dir}\left({ }^{\circ}\right)$ & 267.74 & 231.77 & 35.97 & 146.58 & 0.55 & 0.21 & \\
\hline \multirow{3}{*}{ LEV 2} & Hs (m) & 1.28 & 1.29 & -0.01 & 0.32 & 0.25 & 0.90 & \\
\hline & $\operatorname{Tm}(\mathrm{s})$ & 5.67 & 5.59 & 0.08 & 1.25 & 0.22 & 0.59 & \\
\hline & $\operatorname{Dir}\left({ }^{\circ}\right)$ & 267.74 & 231.61 & 36.13 & 146.75 & 0.55 & 0.21 & \\
\hline \multirow{3}{*}{ LEV 3} & Hs (m) & 1.28 & 1.35 & -0.07 & 0.31 & 0.24 & 0.92 & \\
\hline & $\operatorname{Tm}(\mathrm{s})$ & 5.67 & 5.75 & -0.08 & 1.40 & 0.25 & 0.58 & \\
\hline & $\operatorname{Dir}\left({ }^{\circ}\right)$ & 267.74 & 232.09 & 35.65 & 142.86 & 0.53 & 0.35 & \\
\hline \multirow{2}{*}{ LEV 1} & Hs (m) & 0.78 & 0.90 & -0.12 & 0.32 & 0.42 & 0.78 & \multirow{4}{*}{$\begin{array}{c}\text { B8 Caniçal } \\
(\mathrm{n}=904)\end{array}$} \\
\hline & $\operatorname{Tm}(\mathrm{s})$ & 4.73 & 4.35 & 0.38 & 1.70 & 0.36 & 0.43 & \\
\hline \multirow{2}{*}{ LEV 2} & Hs (m) & 0.78 & 0.64 & 0.14 & 0.27 & 0.35 & 0.84 & \\
\hline & $\operatorname{Tm}(\mathrm{s})$ & 4.73 & 4.03 & 0.70 & 1.56 & 0.33 & 0.37 & \\
\hline
\end{tabular}

using directly the WW3 spectral files and parametric boundary conditions.

The module developed for coastal simulations is illustrated in Fig. 10(a) while the main characteristics of the corresponding computational grids are presented in Table 1. The SWAN coarse area (called also the Madeira wave driver), is defined using spherical coordinates and covers the entire archipelago (level I). This area is connected to the ocean wave forcing with variable boundary conditions provided by the WW3 nodes. Two areas were subsequently defined to cover: Madeira and the Desert Islands, and Porto Santo (level II). At this point the coordinate system was changed to Cartesian and the connection with the driver was made by using again variable boundary conditions. Finally, two higher resolution areas were also coupled using now the standard nesting procedure (level III). These areas were rotated in order to be almost perpendicular to the shore lines. The rotation of the high-resolution grid in Madeira is of 330 degrees whereas the rotation of the high-resolution grid in Porto Santo is of 45 degrees.

The non stationary mode was used always at the levels I and II of the SWAN coastal simulations. For the level III a sequence of stationary simulations was found more effective from the computational point of view with the exception of the most energetic cases when the simulations were performed also in the non stationary mode. As an example, Fig. 10(b) illustrates the wave and wind conditions in Porto Santo (level II) for the time frame 2002/02/02 midday - 12 hours forecast.

Two directional buoys were operating south of Madeira Island as illustrated in Fig. 10(a). These are: Funchal $\left(16.94^{\circ} \mathrm{W}\right.$, $\left.32.62^{\circ} \mathrm{N}\right)$ and Caniçal $\left(16.72^{\circ} \mathrm{W}, 32.72^{\circ} \mathrm{N}\right)$, denoted as B7 and $\mathrm{B} 8$, respectively. An indirect validation scheme was designed by comparing the results of the wave forecast with the measurements. That is why, although the target area was Porto
Santo, simulations have been performed both for Porto Santo and Madeira islands.

Moreover, extended hindcast studies were carried out to check the viability of the wave prediction system. As an example Table 4 illustrates in statistical terms some results for a five-month period. Some further results concerning the wave modeling in Madeira archipelago are presented also in [24].

In parallel, results from the continental coastal environment of Portugal (as presented in Case Study 1) will be also discussed. In this discussion the results from the second level of the SWAN simulations in Madeira will be examined because the utility of the third higher resolution level is disputable when comparing model results with data measured at about 100 meters depth. Moreover, the resolution in the geographical space in this case is enough close with the resolution in the continental simulations considered $\left(0.01^{\circ}\right)$. A first observation resulting from the data analysis in both locations would be that the scheme for the wave generation and propagation developed and focused on the eastern side of the North Atlantic basin provides in general accurate results, especially in terms of significant wave heights. Despite the higher complexity of the physical processes, for the Madeira area the results in terms of bias and RMSE are better than those in the continental Portuguese coastal environment. In Madeira at both buys the absolute biases are less (or around) 0.1 and the RMSE are around 0.3, whereas for the continental nearshore the absolute biases are less than 0.3 and the RMSE between 0.3 and 0.6 . In terms of scatter indices and correlation coefficients the results are sensible equal in both island and continental environments, SI is usually smaller than 0.35 and $r$ is grater than 0.9, with lowest accuracy for B8 in Madeira and better SI for the continental nearshore. In relationship with the wave periods, a general observation would be that the model results are better in terms of mean than peak periods 
systematically. A possible explanation would be that in general mean periods present better the multi peak spectra than peak periods. When comparing the mean periods the results seemed also to be better at B7 in Madeira than in the continental nearshore in almost all parameters, exception the correlation which is about the same value (around 0.7). As expected at B8 in Madeira the correlation is lower (0.36).

However, in terms of mean directions the results are considerably better (with both biases RMSE lower than 10), and well correlated in the continental side (between 0.7 and 0.9 in the northern part) than in the archipelago.

One explanation might be that the local wind surrounding the islands was not appropriately accounted for in the SWAN model simulations and this wind influences more in the wave direction than in the wave heights. Another possible (and more probable) explanation would be that the combined refraction diffraction processes in such complex island environments with very high bathymetric gradients are not yet enough well modeled in the presently operating third generation numerical wave models, unlike the case of the open coasts where the model results in terms of mean direction can be considered acceptable.

\section{Case Study 6 - Operational Forecast in the Coastal Environment Affected by the M/V Prestige Accident}

A second example of operational forecast will be discussed bellow and is presented more extended in [26]. Figure 11(a) ilustrates in background the bathymetric map of the main computational domain corresponding to the SWAN simulations performed in the case of the M/V Prestige accident (North West Iberian nearshore). In foreground Fig. 11(a) presents the wind vectors (white arrows) corresponding to the time frame 2002/12/24h18 (24 hours forecast). The computational strategy is also suggested. Two buoys that were operating in the area, Sillero $\left(9.39^{\circ} \mathrm{W}, 42.1^{\circ} \mathrm{N}\right)$, denoted as B9 and Leixões $(9.09 \mathrm{~W}, 41.2 \mathrm{~N})$, which was denoted as B1 in the first case study, are used as check points and two medium resolution domains were considered in order to increase the resolution closer to the coast. The characteristics of the computational domains are given in Table 1.

For the same time frame (2002/12/24h $18-24$ hours forecast) the wave conditions in the coastal environment affected by the accident of the oil carrier Prestige, are illustrated in Fig. 11(b). In foreground the wave vectors are represented while in background the significant wave height scalar fields are illustrated.

In such cases the Stokes drift may play an important role in driving the oil. In the upper side of Fig. 11(b) the Stokes drift vectors are represented having background the bathymetric map of the northern medium resolution computational domain. The average value of the Stokes velocity, which in that case was $0.17 \mathrm{~m} / \mathrm{s}$, is also represented.

The Stokes drift was estimated using the mass transport relationship given by the second order theory which in deep water leads to [16]:
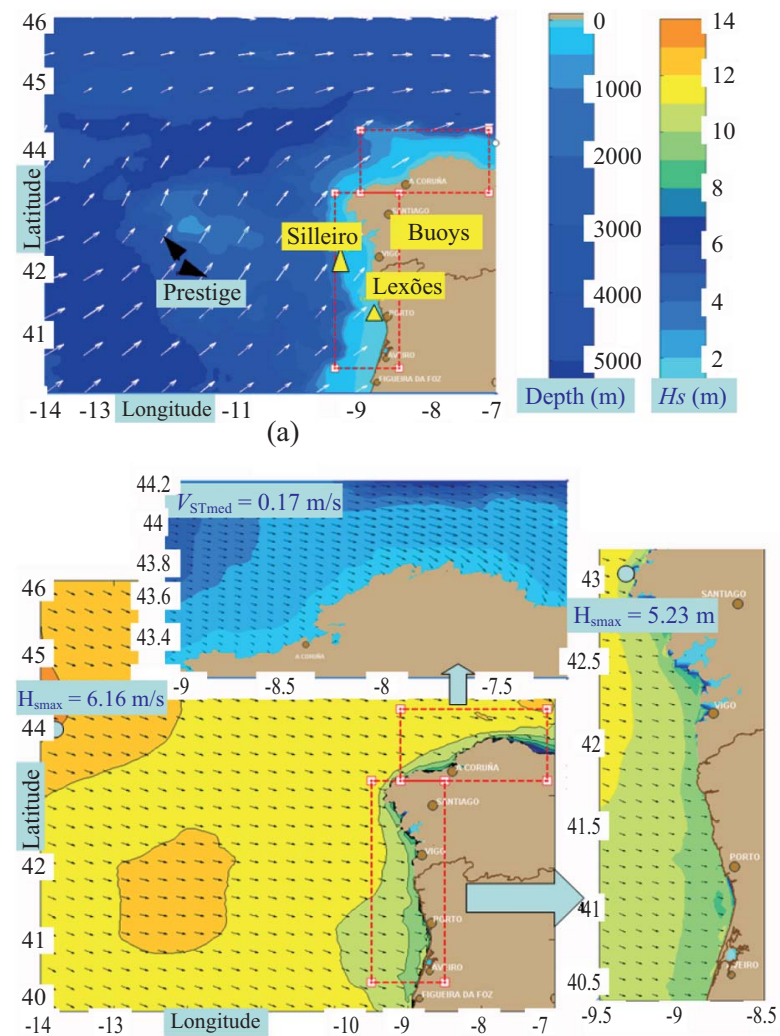

(b)

Fig. 11. Case study 6. (a) Geographical spaces of the SWAN simulations performed in the case of the accident of the Prestige oil carrier. In foreground the wind vectors (white arrows) corresponding to the time frame $2002 / 12 / 24 h 18$ ( 24 hours forecast) are illustrated, while in background the bathymetric map. Two buoys that were operating in the area are also represented. (b) Wave conditions in the coastal environment affected by the accident of the oil carrier Prestige, time frame 2002/12/24h18 (24 hours forecast). In foreground the wave vectors are represented while in background the significant wave height scalar fields are illustrated. In the upper side of the figure the component induced by the Stokes drift to the oil spill velocity is represented with black arrows having in background the bathymetric map.

$$
u_{s}(z)=\left(\frac{\pi H}{L}\right)^{2} \cdot c \cdot \exp \left(\frac{4 \pi z}{L}\right)
$$

in which $H$ is the wave height, $c$ is the relative phase velocity and $z$ represents the vertical coordinate of the water particle measured from the quiet free surface. Since the goal was to estimate an average value of the Stokes drift at the surface, it was considered $z=0$ and the root mean square wave height $\left(H_{r m s}\right)$ was used instead of $H$ in relationship (21). $H_{r m s}$ was deduced from the significant wave height considering the standard Rayleigh distribution.

Hence, in the case of an irrotational motion the mass transport velocity leads to a slow drift in direction of the wave motion (on the order of $\mathrm{ka}^{2}$, where $k$ is the wave number and $a$ 
the wave amplitude). Moreover, when the water surface is contaminated by an inextensible slick a surface streaming relative to the fluid bellow will be induced due to molecular viscosity. Philips [18] showed that in deep water the oil slick streams ahead the fluid particles at the surface by a velocity excess of:

$$
\Delta u_{s}=\frac{3}{4}\left(\frac{\pi H}{L}\right)^{2} \cdot c .
$$

It means that a patch of slick move faster than the surrounding clean surface, the total velocity being the sum of (19) and the Lagrangian drift in deep water (20), or seven-fourths the classical result of Stokes. Consequently the wave induced drift driving the pollution in the sea that should be added to the others components of the surface currents, should be evaluated with the relationship bellow:

$$
U_{\text {Stokes }}=u_{s}+\Delta u_{s}=\frac{7}{4}\left(\frac{\pi H}{L}\right)^{2} \cdot c .
$$

Finally some results concerning the reliability of the wave forecast in the coastal environment affected by the $\mathrm{M} / \mathrm{V}$ Prestige accident are presented in Fig. 12(a) that illustrates a direct comparison between the simulated and the measured results at the buoy of Leixões for the main wave parameters (significant wave height, period and direction) in December 2002. Three different cases were considered: nowcast, 12 hours forecast and 24 hours forecast, respectively. The data presented refer to the same time moment of the day (midday). Fig. 12(b) presents the relative errors corresponding to the same situations. The corresponding statistical results are presented in Table 5.

\section{CONCLUDING REMARKS}

Starting with 1960s, when the wave models from the first generation became operational, the spectral models developed to the actual stage of third generation models.

These are full spectral models based on solving the action balance equation in all five dimensions (time, geographical and spectral spaces) with source terms accounting for the most relevant physical processes in both deep and shallow water. In the last decade the wave models became effective tools in operational oceanography and they are providing reliable wave predictions at various scales.

As an attempt to present a more complete picture of the modeling process, the present work considers six different case studies. The first two are of hindcast type, the next two are nowcasts and finally the last two refer to situations of operational forecast. Generally speaking the highest precision of the wave models should be in the nowcast schemes while the forecast products present the greatest degree of incertitude.

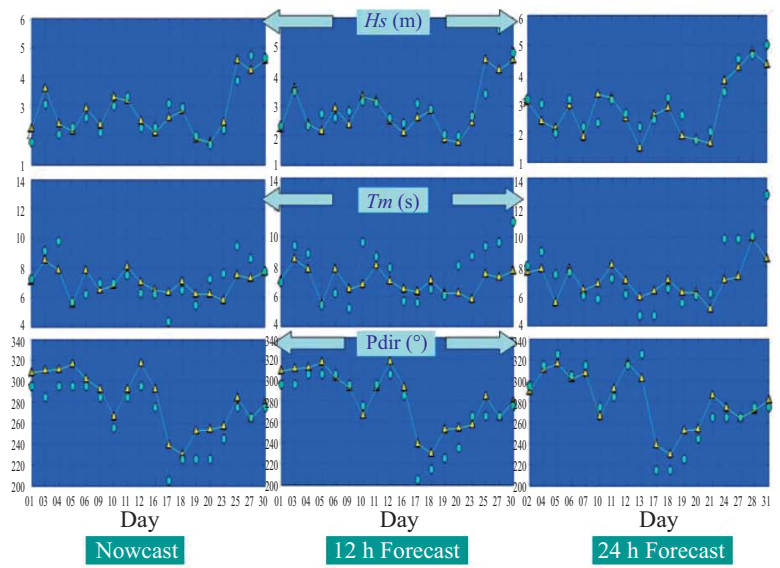

(a)

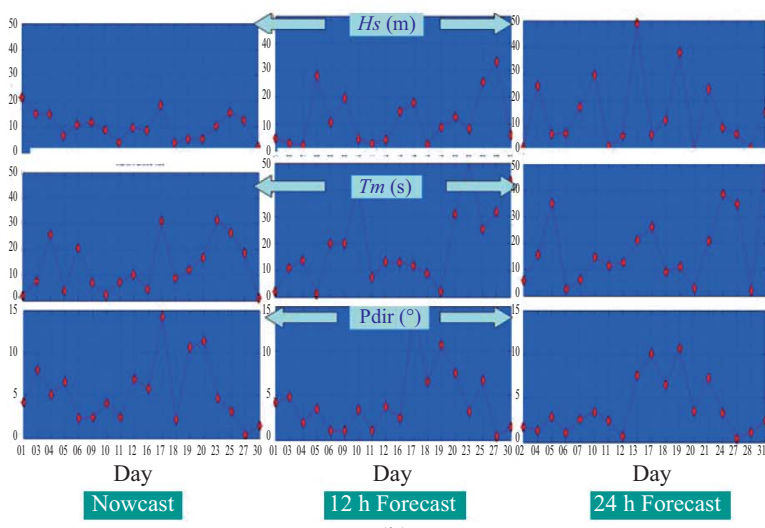

(b)

Fig. 12. Case study 6. Wave forecast in the coastal environment affected by the M/V Prestige accident. (a) Direct comparison between the simulated and the corresponding results measured at Leixões buoy for the significant wave height, period and direction, December 2002. (b) Relative errors of the simulations results against the corresponding data measured at $\mathrm{B} 1$, for the significant wave height, period and direction, December 2002. Three different cases were considered: nowcast, 12 hours forecast and 24 hours forecast, respectively.

In order to give a better perspective of the model performances, the hindcast studies are in general performed for long periods of time, at least several months, while the time periods considered for the nowcast schemes discussed herewith are of only a few days. The time window for the wave predictions in the examples of operational forecast discussed was of maximum three days.

As regards the hindcast studies, in the west Iberian nearshore a wave prediction system based on the interaction between the two state of the art spectral wave models (WAM and SWAN) was used while for the Black Sea basin the wave prediction system was entirely SWAN based. In both nowcast schemes discussed in the present work, SHORECIRC modeling system was connected to the wave prediction systems. SHORECIRC has REFDIF as wave driver, which is a phase-resolving model based on the mild slope equation, and also a quasi $3 \mathrm{D}$ circulation part that allows the assessment of 
Table 5. Case study 6. Statistical results for the wave forecast performed in the case of the accident of the oil carrier Prestige (simulations for December 2002). B1 - Leixões, B9 - Sillero.

\begin{tabular}{|c|c|c|c|c|c|c|}
\hline Prediction type & Wave parameter & $\mathrm{B}_{\mathrm{med}}$ & $\mathrm{S}_{\mathrm{med}}$ & Bias & RMSE & Buoy \\
\hline \multirow{3}{*}{ Nowcast } & Hs (m) & 2.86 & 2.77 & 0.09 & 0.34 & \multirow{9}{*}{$\begin{array}{c}\text { B1 } \\
\text { Leixões }\end{array}$} \\
\hline & $\operatorname{Tm}(\mathrm{s})$ & 6.98 & 7.17 & -0.19 & 1.14 & \\
\hline & $\operatorname{Dir}\left({ }^{\circ}\right)$ & 281.39 & 266.67 & 14.72 & 17.52 & \\
\hline \multirow{3}{*}{$\begin{array}{c}\text { Forecast } \\
12 \mathrm{H}\end{array}$} & Hs (m) & 2.86 & 3.00 & -0.14 & 0.50 & \\
\hline & $\operatorname{Tm}(\mathrm{s})$ & 6.98 & 7.72 & -0.74 & 1.66 & \\
\hline & $\operatorname{Dir}\left({ }^{\circ}\right)$ & 281.39 & 272.78 & 8.61 & 14.46 & \\
\hline \multirow{3}{*}{$\begin{array}{c}\text { Forecast } \\
24 \mathrm{H}\end{array}$} & Hs (m) & 2.81 & 2.95 & -0.14 & 0.44 & \\
\hline & $\operatorname{Tm}(\mathrm{s})$ & 7.03 & 7.40 & -0.37 & 1.62 & \\
\hline & $\operatorname{Dir}\left({ }^{\circ}\right)$ & 280.88 & 277.78 & 3.1 & 13.0 & \\
\hline \multirow{2}{*}{ Nowcast } & Hs (m) & 3.34 & 3.39 & -0.05 & 0.50 & \multirow{6}{*}{$\begin{array}{c}\text { B9 } \\
\text { Sillero }\end{array}$} \\
\hline & $\operatorname{Tm}(\mathrm{s})$ & 7.72 & 7.49 & 0.23 & 1.30 & \\
\hline \multirow{2}{*}{$\begin{array}{c}\text { Forecast } \\
12 \mathrm{H}\end{array}$} & Hs (m) & 3.34 & 3.57 & -0.23 & 0.54 & \\
\hline & $\operatorname{Tm}(\mathrm{s})$ & 7.72 & 7.87 & -0.15 & 0.86 & \\
\hline \multirow{2}{*}{$\begin{array}{c}\text { Forecast } \\
24 \mathrm{H}\end{array}$} & Hs (m) & 3.16 & 3.33 & -0.17 & 0.45 & \\
\hline & $\operatorname{Tm}(\mathrm{s})$ & 7.32 & 7.31 & 0.01 & 1.28 & \\
\hline
\end{tabular}

the coastal circulation. In the two forecast examples presented, the offshore wave forcing was provided by WW3 model run by FNMOC (acronym from Fleet Numerical Meteorology and Oceanography Center) that is well calibrated and tested over the entire North Atlantic Ocean.

In order to increase the model resolution in the nearshore direction SWAN was nested in itself several times. Some analysis in spatial frames illustrated the coastal focusing of the modeling systems as for example the cases presented in Fig. 2 and 6 , respectively. Besides significant wave heights fields and wave vectors, the spatial distribution of some additional parameters, either scalar or vectors can be analyzed after case as for example the wave energy presented in Fig. 3 and the Stokes drift illustrated in Fig. 11(b).

Wave models can fail in their predictions from various reasons. Some most probable would be: inaccurate wind data, inadequate model parameterisation, weak representation inside the model of complex physical processes, numerical effects induced by limitations of the computational scheme and also human errors in data manipulation or in the modelling process. Hence a key issue when modelling waves would be to be able to identify properly when such situation may occur and how to avoid it.

In relationship with the wind field, as it was previously mentioned, the atmospheric models usually provide reliable forecast for a time window of a few days. Hence wave forecasts exceeding this interval can be considered rather unrealistic. In relationship with the measured wind an important issue that should be accounted for is that the wave models deal with the wind at 10 meters height, so if the height is different it should be adjusted with relationships as for example that given by [12].
SWAN model is very flexible and can be applied for a wide range of coastal applications. The default model parameterization is the simplest way but it is not automatically the most appropriate approach in wave modeling. Hence a key issue would be to assess the most appropriate model configuration. This depends on the specific site and on the characteristics and the dynamics of the environmental matrix.

As the present work fully illustrated whitecapping is obviously the weak link in deep water wave modeling. Once the physical parameterization for a certain process (in this case whitecapping) is chosen the next step would be to find the most appropriate values for the tunable parameters. In order to do this, calibration studies in both space and time frames should be performed.

A very important aspect is to be able to perform a correct identification of most relevant processes that affect a specific geographical domain in certain conditions. For example if wave current interactions are relevant in either offshore or nearshore the wave model should be coupled with a circulation model and run in an iterative manner. This is illustrated by the case studies III and IV when the wave induced nearshore currents were evaluated using SHORECIRC model system. In the case study VI the Stokes drift was relevant and this was estimated using the results from the second order theory.

Another important aspect is related with the accuracy and resolution of the bathymetric data. As case study IV fully illustrated, high gradients in the bathymetry and accentuated 3D characteristics might decrease the SWAN model performances in the nearshore.

A final general conclusion coming from the present work would be that, under certain limitations, the spectral wave 
models developed to the actual stage of third generation account for the most relevant physical processes in both deep and shallow water. From these reasons, in the last decade the wave models became effective tools in operational oceanography and they are providing reliable wave predictions at various scales. Moreover, at least as regards SWAN, its dynamics is very high and new improvements are implemented almost every year in the model.

\section{REFERENCES}

1. Alves, J. H. G. M. and Banner, M. L., "Performance of a saturation-based dissipation-rate source term in modelling the fetch-limited evolution of wind waves," Journal of Physical Oceanography, Vol. 33, pp. 1274-1298 (2003).

2. Athanassoulis, G. A., Belibassakis, K., A. and Gerostathis, Th., "The POSEIDON nearshore wave model and its application to the prediction of the wave conditions in the nearshore/coastal regions of the Greek Seas,' Global Atmosphere and Ocean System (GAOS), Vol. 8, No. 2-3, pp. 101117 (2002).

3. Booij, N. and Holthuijsen, L. H., "Propagation of ocean waves in discrete spectral wave models," Journal of Computational Physics, Vol. 68, pp. 307-326 (1987).

4. Booij, N., Ris, R. C., and Holthuijsen, L. H., "A third generation wave model for coastal regions, Part 1: Model description and validation," Journal of Geophysical Research, Vol. 104, No. C4, pp. 7649-7666 (1999).

5. Coli, A., Conley, D. C., Santos, J. A., and Pires-Silva, A., "Estimation of offshore directional spectra by inverse methodology at Porto Ferro, Sardinia," Proceedings of the $17^{\text {th }}$ International Offshore and Polar Engineering Conference, Lisbon, ISOPE-2007-JSC-535, 7p (2007).

6. Gerostathis, Th., Belibassakis, K., A., and Athanassoulis, G., A., "A coupled-mode, phase-resolving model for the transformation of wave spectrum over steep 3D topography: parallel-architecture implementation,' Journal of Offshore Mechanics and Arctic Engineering, Vol. 130, No.1, pp. 389-397 (2008).

7. Gomes, F., Pacheco, L.B., Silva, J., Silva, R., and Rusu, E., Using GIS In The Evaluation of the Wave Induced Circulation in the Portuguese Nearshore, EGS General Assembly, Nice, France (2004).

8. Gómez, M. and Carretero, J. C., "A two-way nesting procedure for the WAM model: application to the Spanish coast," Journal of Offshore Mechanics and Arctic Engineering, Vol. 119, pp. 20-24 (1997).

9. Guedes Soares, C., Weisse, R., Carretero J. C., and Alvarez, E., "A 40 years hindcast of wind, sea level and waves in European waters," Proceedings of the 21 st International Conference on Offshore Mechanics and Arctic Engineering (OMAE'02), Oslo, Norway, Paper no. OMAE200228604, pp. 669-675 (2002).

10. Hasselmann, K., "On the spectral dissipation of ocean waves due to white-cap," Boundary-Layer Meteorology, Vol. 6, No. 1-2, pp. 107-127 (1974).

11. Holthuijsen, L. H., Herman, A., and Booij, N., "Phase-decoupled refractiondi difraction for spectral wave models," Coastal Engineering, Vol. 49, pp. 291-305 (2003).

12. Hsu, S. A., Meindl, E. A., and Gilhousen, D., "Determining the powerlaw wind-profile exponent under near-neutral stability conditions at sea," Journal of Applied Meteorology, Vol. 33, No. 6, pp. 757-765 (1994).

13. Janssen, P. A. E. M., "Quasi-linear theory of wind-wave generation applied to wave forecasting," Journal of Physical Oceanography, Vol. 21, pp. 1631-1642 (1991).

14. Jonsson, I. G.., "Wave-current interactions," In: LeMehaute, B. and Hanes, D. M. (Ed.), The sea, John Wiley \& Sons, Inc., New York, Vol. 9, Part A, B, pp. 65-120 (1990).

15. Komen, G. J., Hasselmann, S., and Hasselmann, K., "On the existence of a fully developed wind sea spectrum," Journal of Physical Oceanography, Vol. 14, pp. 1271-1285 (1984).

16. Lakshmi, H. K. and Clayson, C. A., Small Scale Processes in Geophysical Fluid Flows, AP-International Geophysics Series, Academic Press, San Diego, California, Vol. 67, 888 p (2000).

17. Mettlach, T. R., Earle, M. D., and Hsu, Y. L., Software Design Document for the Navy Standard Surf Model, Version 3.2, Naval Research Laboratory, Stennis Space Center, Mississippi, 187p (2002).

18. Phillips, O. M., The Dynamics of Upper Ocean, Cambridge University Press, Cambridge (1977).

19. Rogers, W. E., Kaihatu, J. M., Petit, H. A. H., Booij, N., and Holthuijsen, L. H., "Diffusion reduction in a arbitrary scale third generation wind wave model," Ocean Engineering, Vol. 29, No. 11, pp. 1357-1390 (2002).

20. Rusu, E., "Wave energy assessments in the Black Sea," Journal of Marine Science and Technology, Vol. 14, No. 3, pp. 359-372 (2009).

21. Rusu, E., Conley, D. C., and Coelho, E. F., "A hybrid framework for predicting waves and longshore currents," Journal of Marine Systems, Vol. 69, pp. 59-73 (2008)

22. Rusu, E. and Guedes Soares, C., "Numerical modeling to estimate the spatial distribution of the wave energy in the Portuguese nearshore," Renewable Energy, Vol. 34, No. 6, pp. 1501-1516 (2009).

23. Rusu, E. and Guedes Soares, C., "Validation of two wave and nearshore current models at three field sites," Journal of Waterway, Port, Coastal, and Ocean Engineering, Vol. 136, No. 1, pp. 27-45 (2010).

24. Rusu, E., Pilar, P., and Guedes Soares, C., "Evaluation of the Wave Conditions in Madeira Archipelago with Spectral Models," Ocean Engineering, Vol. 35, issue 13, pp. 1357-1371 (2008).

25. Rusu, E., Pinto, J. P., Silva, R., and Soares, C. V., "A method to predict wave conditions in island environment," The $7^{\text {th }}$ International Workshop on Wave Hindcasting and Forecasting, Banff, Alberta, Canada, pp. 215226 (2002).

26. Rusu, E., Silva, R., Soares, C. V., and Rusu, L., "Wave forecast in the coastal environment affected by M/V Prestige breakdown," Thalassas International Journal of Marine Science, Vol. 2, pp. 161-162 (2003).

27. Silva, R., Jorge da Silva, A., Rusu, E., Oliveira, F., Larangeiro, S., and Taborda R., "Evaluation of the longshore current for a sector of the Portuguese west coast: Application of different methodologies," Coastal Engineering 2004, World Scientific Pub Co Inc 2005, Vol. 2, pp. 14551467 (2004).

28. Smith, J. M., Sherlock, A. R., and Resio, D. T., STWAVE: Steady-State Spectral Wave Model, user's guide for STWAVE, Version 3.0, ERDC/CHL SR-01-01, U.S. Army Engineer Research and Development Center, Vicksburg, MS (2001).

29. Stelling, G. S. and Leendertse, J. J., "Approximation of convective processes by cyclic AOI methods," Proceeding of the 2nd International Conference on Estuarine and Coastal Modeling, ASCE Tampa, Florida, pp. 771-782 (1992).

30. Svendsen, I. A., Haas, K., and Zhao, Q., Quasi-3D Nearshore Circulation Model SHORECIRC, version 2.0, Center for Applied Coastal Research, University of Delaware, Newark, DE 19716 U.S.A. (2002).

31. Tolman, H. L., "A third-generation model for wind waves on slowly varying, unsteady and inhomogeneous depths and currents," Journal of Physical Oceanography, Vol. 21, No. 6, pp. $782-797$ (1991).

32. Van Vledder, G. Ph. and Hurdle, D. P., "Performance of formulations for whitecapping in wave prediction models," Proceedings of the 21st International Conference on Offshore Mechanics and Arctic Engineering (OMAE'02), Oslo, Norway, Paper no. OMAE2002-28146, pp. 155-163 (2002).

33. WAMDI group, "The WAM model - a third generation ocean wave predict-tion model," Journal of Physical Oceanography, Vol. 18, pp 17751810 (1988).

34. Yan, L., "An improved wind input source term for third generation ocean wave modeling," Scientific report WR-No 87-8, De Bilt, The Netherlands (1987). 